\title{
Robust Estimation of Causal Effects via High-Dimensional Covariate Balancing Propensity Score
}

\author{
Yang Ning* Sida Peng ${ }^{\dagger} \quad$ Kosuke Imai ${ }^{\ddagger}$
}

\begin{abstract}
In this paper, we propose a robust method to estimate the average treatment effects in observational studies when the number of potential confounders is possibly much greater than the sample size. We first use a class of penalized $M$-estimators for the propensity score and outcome models. We then calibrate the initial estimate of the propensity score by balancing a carefully selected subset of covariates that are predictive of the outcome. Finally, the estimated propensity score is used to construct the inverse probability weighting estimator. We prove that the proposed estimator, which has the sample boundedness property, is root- $n$ consistent, asymptotically normal, and semiparametrically efficient when the propensity score model is correctly specified and the outcome model is linear in covariates. More importantly, we show that our estimator remains root- $n$ consistent and asymptotically normal so long as either the propensity score model or the outcome model is correctly specified. We provide valid confidence intervals in both cases and further extend these results to the case where the outcome model is a generalized linear model. In simulation studies, we find that the proposed methodology often estimates the average treatment effect more accurately than the existing methods. We also present an empirical application, in which we estimate the average causal effect of college attendance on adulthood political participation. Open-source software is available for implementing the proposed methodology. ${ }^{1}$
\end{abstract}

Keyword: causal inference, double robustness, model misspecification, post-regularization inference, semiparametric efficiency

\section{Introduction}

Propensity score of Rosenbaum and Rubin (1983) plays a central role in the estimation of causal effects in observational studies (see e.g., Imbens, 2000; Imai and van Dyk, 2004, for extensions to a

\footnotetext{
${ }^{*}$ Department of Statistical Science, Cornell University, Ithaca, New York 14853, U.S.A. ; e-mail: yn265@cornell.edu.

${ }^{\dagger}$ Department of Economics, Cornell University, Ithaca, New York 14853, U.S.A. ; e-mail: sp947@cornell.edu.

${ }^{\ddagger}$ Department of Government and Department of Statistics, Harvard University, Cambridge, Massachusetts 02138, U.S.A. e-mail: imai@harvard.edu.

${ }^{1}$ An earlier version of this paper available online at June 2017 has a different title "High Dimensional Propensity Score Estimation via Covariate Balancing".
} 
non-binary treatment). In particular, matching and weighting methods based on propensity score have become part of applied researchers' standard toolkit across many scientific disciplines (see e.g., Lunceford and Davidian, 2004; Rubin, 2006). One important challenge, which is becoming increasingly common as the amount of available data grows, is the question of how to incorporate a large number of potential confounders. For example, Schneeweiss et al. (2009) considers a total of several thousand candidate confounders obtained from the health care claims data.

In this paper, we propose a robust method to estimate the average treatment effect (ATE) in observational studies when the number of potential confounders is possibly much greater than the sample size. In particular, under the standard assumption of strong ignorability, we propose to estimate the propensity score by balancing covariates in high-dimensional settings. The proposed method consists of several steps. We first obtain an initial estimator of the propensity score model by maximizing a penalized generalized quasi-likelihood, which depends on a user-specified weight function. Next, we apply the weighted least squares method to fit the outcome model. We show that the two weight functions critically determine the performance of the proposed estimator under model misspecification. Third, we refine the initial estimate of the propensity score by balancing a carefully selected set of observed covariates that are predictive of the outcome. Finally, the estimated propensity score is used to construct the inverse probability weighting estimator of the ATE.

We prove that under mild conditions the proposed estimator of ATE is root- $n$ consistent, asymptotically normal, and semiparametrically efficient, when the propensity score model is correctly specified and the outcome model is linear in covariates. This result holds for a broad class of weight functions used in the initial estimation of propensity score. However, the proposed estimator typically has a slower rate of convergence under misspecified models. To address this problem, we show that by carefully choosing the weight functions the proposed estimator remains root- $n$ consistent and asymptotically normal so long as either the propensity score model or the outcome model is correctly specified. The proposed estimator has the double robustness and sample boundedness properties and comes with honest confidence intervals. Finally, we extend these theoretical results to the case where the outcome model is a generalized linear model in order to allow for nonlinearity.

We emphasize that the proposed methodology does not require the variable selection consistency of either the propensity score model or the outcome model. This is because our goal is to estimate causal effects rather than the coefficients of propensity score and outcome models. The covariate balancing step of our methodology removes the bias that results from the failure to select some covariates.

The proposed methodology, which we call the high-dimensional covariate balancing propensity score (HD-CBPS), builds on three strands of research that have recently emerged in the causal inference literature. In the following, we briefly highlight the differences between the HD-CBPS and the existing methods. Section 3.5 further presents a more detailed comparison.

First, a number of researchers have recently proposed to estimate the ATE by optimizing covariate balance between the treatment and control groups (e.g., Hainmueller, 2012; Graham et al., 2012; Imai and Ratkovic, 2014; Chan et al., 2016; Zubizarreta, 2015; Zhao, 2016; Fan et al., 2016). 
It has been shown, both theoretically and empirically, that these approaches can significantly improve the efficiency and robustness of standard propensity score methods. The proposed HD-CBPS methodology extends the covariate balancing propensity score (CBPS) methodology of Imai and Ratkovic (2014) and Fan et al. (2016) to the high-dimensional settings, in which the number of potential confounders is possibly greater than the sample size. While the original CBPS methodology estimates the propensity score by balancing all covariates, this is not an effective strategy in high-dimensional settings because the number of covariates is too large. To address this issue, we propose a weak covariate balancing approach, which yields a root- $n$ consistent estimator in high-dimensional settings.

Second, we contribute to the growing literature on the estimation of the ATE in high-dimensional settings. Belloni et al. (2014) proposed a double selection approach to infer the coefficient of treatment variable in a partially linear model under the assumption that both the outcome and treatment models are sparse. Farrell (2015), Belloni et al. (2013a), and Chernozhukov et al. (2016) extended the augmented inverse probability weighting (AIPW) estimator of Robins et al. (1994) to high-dimensional settings. A common characteristic of these methods is to first estimate the nuisance parameters (e.g., propensity score) typically by the penalized maximum likelihood and then estimate the ATE by solving the efficient score function. Different from this line of work, we rely on the covariate balancing strategy for estimating the propensity score model and use the Horvitz-Thompson estimator (Horvitz and Thompson, 1952) for inferring the ATE without employing the AIPW estimator. As elaborated in Section 3.5, the robustness of the asymptotic distributions of our estimator to model misspecification is the main advantage over these existing methods. Most recently, Tan $(2017,2018)$ proposed a penalized calibrated propensity score method and studied its robustness to model misspecification. Unlike this method, our approach is based on the covariate balancing. See Section 3.5 for a detailed comparison.

Finally, HD-CBPS is related to the recently proposed approximate residual balancing method (Athey et al., 2016), which unlike our methodology has an advantage of not requiring the formulation of a propensity score model. While the approximate residual balancing method requires the outcome model to be linear in covariates, HD-CBPS can yield a consistent and asymptotically normal estimator even under the misspecification of the outcome model so long as the propensity score is correctly specified. In addition, we show that the proposed method and its asymptotic theory can be extended to the case, in which the outcome variable follows a generalized linear model. This also overcomes the same limitation of the original CBPS estimator (Imai and Ratkovic, 2014; Fan et al., 2016). Finally, we also argue that the estimation of the propensity score may help scientists better understand the treatment assignment mechanism (e.g., Rubin, 2008), when it is correctly specified. Open-source software package, CBPS, is available for implementing our proposed methodology (Fong et al., 2018).

Throughout the paper, we use the following notation. For $v=\left(v_{1}, \ldots, v_{d}\right)^{\top} \in \mathbb{R}^{d}$, and $1 \leq q \leq \infty$, we define $\|v\|_{q}=\left(\sum_{i=1}^{d}\left|v_{i}\right|^{q}\right)^{1 / q},\|v\|_{0}=|\operatorname{supp}(v)|$, where $\operatorname{supp}(v)=\left\{j: v_{j} \neq 0\right\}$ and $|A|$ is the cardinality of a set $A$. Denote $v^{\otimes 2}=v v^{\top}$. If the matrix $M$ is symmetric, then $\lambda_{\min }(M)$ and $\lambda_{\max }(M)$ are the minimal and maximal eigenvalues of $M$. For $S \subseteq\{1, \ldots, d\}$, let $v_{S}=\left\{v_{j}: j \in S\right\}$ and $S^{c}$ be the complement of $S$. For two positive sequences $a_{n}$ and $b_{n}$, we write $a_{n} \asymp b_{n}$ if 
$C \leq a_{n} / b_{n} \leq C^{\prime}$ for some $C, C^{\prime}>0$. Similarly, we use $a_{n} \lesssim b_{n}$ to denote $a_{n} \leq C b_{n}$ for some constant $C>0$. A random variable $X$ is sub-exponential if there exists some constant $K_{1}>0$ such that $\mathbb{P}(|X|>t) \leq \exp \left(1-t / K_{1}\right)$ for all $t \geq 0$. The sub-exponential norm of $X$ is defined as $\|X\|_{\psi_{1}}=\sup _{p \geq 1} p^{-1}\left(\mathbb{E}|X|^{p}\right)^{1 / p}$. A random variable $X$ is sub-Gaussian if there exists some constant $K_{2}>0$ such that $\mathbb{P}(|X|>t) \leq \exp \left(1-t^{2} / K_{2}^{2}\right)$ for all $t \geq 0$. The sub-Gaussian norm of $X$ is defined as $\|X\|_{\psi_{2}}=\sup _{p \geq 1} p^{-1 / 2}\left(\mathbb{E}|X|^{p}\right)^{1 / p}$. Denote $a \vee b=\max (a, b)$.

\section{The Proposed Methodology}

\subsection{Setup}

Suppose that we observe a simple random sample of size $n$ from a population of interest. For each unit $i$, we observe a 3 -tuple $\left(T_{i}, Y_{i}, X_{i}\right)$ where $X_{i}$ is a $d$-dimensional vector of pre-treatment covariates, $Y_{i}$ is an outcome variable, and $T_{i}$ is a binary treatment variable denoting whether the observation receives the treatment $\left(T_{i}=1\right)$ or not $\left(T_{i}=0\right)$. Let $Y_{i}(1)$ and $Y_{i}(0)$ denote the potential outcomes under the treatment and control conditions, respectively. This notation implies the stable unit treatment value assumption (Rubin, 1990). Then, the observed outcome can be written as $Y_{i}=Y_{i}\left(T_{i}\right)$. Our goal is to infer the average treatment effect (ATE),

$$
\mu^{*}=\mathbb{E}\left\{Y_{i}(1)-Y_{i}(0)\right\}
$$

We focus on the estimation of $\mu_{1}^{*}=\mathbb{E}\left\{Y_{i}(1)\right\}$ since $\mu_{0}^{*}=\mathbb{E}\left\{Y_{i}(0)\right\}$ can be estimated in a similar manner. We impose a working parametric model $\pi\left(X_{i}^{\top} \beta\right)$ for the treatment assignment mechanism, which is known as the propensity score $\mathbb{P}\left(T_{i}=1 \mid X_{i}\right)$, where $\pi(\cdot)$ is a known function and $\beta$ is an unknown $d$-dimensional vector. In this work, we consider the settings where the number of covariates is possibly much greater than the sample size, i.e., $d \gg n$. When the propensity score model is correctly specified, we have

$$
\mathbb{P}\left(T_{i}=1 \mid X_{i}\right)=\pi\left(X_{i}^{\top} \beta^{*}\right)
$$

for some $\beta^{*} \in \mathbb{R}^{d}$. Similarly, for the outcome variable we impose a linear working model. When the working model is correctly specified, we have

$$
\mathbb{E}\left\{Y_{i}(1) \mid X_{i}\right\}=K_{1}\left(X_{i}\right)
$$

where $K_{1}\left(X_{i}\right)=\alpha^{* \top} X_{i}$ for some $\alpha^{*} \in \mathbb{R}^{d}$. An extension to the generalized linear models will be studied in Section 4. In general, the propensity score model (2.2) or the outcome model (2.3) can be misspecified. We begin by assuming both models (2.2) and (2.3) hold. When studying theoretical properties of our proposed methodology in Section 3, however, we will consider the situations, in which either model (2.2) or (2.3) does not hold. 


\subsection{High-Dimensional Covariate Balancing Propensity Score}

In many applications, it is often reasonable to assume that the propensity score model is sparse or approximately sparse. Under the sparsity assumption, Tibshirani (1996) and Fan and Li (2001) proposed the penalized maximum likelihood estimators (PMLEs) for parameter estimation and prediction. Unfortunately, the PMLE cannot be directly used with the Horvitz-Thompson estimator to infer $\mu_{1}^{*}=\mathbb{E}\left\{Y_{i}(1)\right\}$ because the PMLE may incur a large bias due to shrinkage and its limiting distribution is often non-normal. Thus, the resulting estimator may have a slower rate of convergence and an intractable limiting distribution.

To address this problem, we estimate the propensity score by optimizing covariate balance between the treatment and control groups. To this end, we distinguish the following two types of covariate balancing properties.

Definition 2.1 (Covariate Balancing Properties). Let $\widehat{\pi}=\pi\left(X^{\top} \widehat{\beta}\right)$ denote an estimator of the propensity score $\mathbb{P}(T=1 \mid X)$ with $\widehat{\beta}$ being an estimator of $\beta^{*}$, which is the true value of $\beta$.

(a) We call $\widehat{\pi}$ satisfies the strong covariate balancing property if the following equality holds,

$$
\sum_{i=1}^{n}\left(\frac{T_{i}}{\widehat{\pi}_{i}}-1\right) X_{i}=0 .
$$

(b) We call $\widehat{\pi}$ satisfies the weak covariate balancing property if the following equality holds,

$$
\sum_{i=1}^{n}\left(\frac{T_{i}}{\widehat{\pi}_{i}}-1\right) \alpha^{* \top} X_{i}=0
$$

where $\alpha^{*}$ is defined by $K_{1}\left(X_{i}\right)=\alpha^{* \top} X_{i}$ in equation $(2.3)$.

Although the strong covariate balancing property implies the weak one, the converse does not necessarily hold. The existing covariate balancing propensity score methods aim to achieve the strong covariate balancing property, which balances the mean of every component of $X_{i}$ (e.g., Imai and Ratkovic, 2014; Fan et al., 2016). However, constructing an estimator $\widehat{\pi}$ with the strong covariate balancing property is difficult in high-dimensional settings. When $d>n$, the estimator $\widehat{\beta}$ that satisfies equation (2.4) is not unique and therefore not even well defined. In addition, imposing additional penalty or constraint may introduce bias because it may not satisfy the strong covariate balancing property.

To overcome this difficulty, we propose to estimate the propensity score such that equation (2.5) rather than equation (2.4) approximately holds. We show that the weak covariate balancing property is sufficient to remove the bias from the estimation of the propensity score model. Here, we first introduce the proposed methodology, which we call the high-dimensional covariate balancing propensity score (HD-CBPS). 
Step 1: Define a generalized quasi-likelihood function as

$$
Q_{n}(\beta)=\frac{1}{n} \sum_{i=1}^{n} \int_{0}^{\beta^{\top} X_{i}}\left\{\frac{T_{i}}{\pi(u)}-1\right\} w_{1}(u) d u,
$$

where $w_{1}(\cdot)$ is a positive weight function. Compute the regularized estimator

$$
\widehat{\beta}=\underset{\beta \in \mathbb{R}^{d}}{\operatorname{argmin}}-Q_{n}(\beta)+\lambda\|\beta\|_{1},
$$

where $\lambda>0$ is a tuning parameter.

Step 2: Define a weighted least square loss function using the treatment group as

$$
L_{n}(\alpha)=\frac{1}{n} \sum_{i=1}^{n} T_{i} w_{2}\left(\widehat{\beta}^{\top} X_{i}\right)\left(Y_{i}-\alpha^{\top} X_{i}\right)^{2}
$$

where $w_{2}(\cdot)$ is another positive weight function. Compute the regularized estimator

$$
\widetilde{\alpha}=\underset{\alpha \in \mathbb{R}^{d}}{\operatorname{argmin}} L_{n}(\alpha)+\lambda^{\prime}\|\alpha\|_{1}
$$

where $\lambda^{\prime}>0$ is a tuning parameter.

Step 3: Let $\widetilde{S}=\left\{j:\left|\widetilde{\alpha}_{j}\right|>0\right\}$ denote the support of $\widetilde{\alpha}$ and $X_{\widetilde{S}}$ denote the corresponding subset of $X$. We calibrate the initial estimator $\widehat{\beta}_{\widetilde{S}}$ to balance $X_{\widetilde{S}}$. Specifically, we solve,

$$
\widetilde{\gamma}=\underset{\gamma \in \mathbb{R}|\widetilde{S}|}{\operatorname{argmin}}\left\|g_{n}(\gamma)\right\|_{2}^{2} \text { where } g_{n}(\gamma)=\frac{1}{n} \sum_{i=1}^{n}\left\{\frac{T_{i}}{\pi\left(\gamma^{\top} X_{i \widetilde{S}}+\widehat{\beta}_{\widetilde{S}^{c}}^{\top} X_{i \widetilde{S}^{c}}\right)}-1\right\} X_{i \widetilde{S}}
$$

We then set $\widetilde{\beta}=\left(\widetilde{\gamma}, \widehat{\beta}_{\widetilde{S}^{c}}\right)$ and $\widetilde{\pi}_{i}=\pi\left(\widetilde{\beta}^{\top} X_{i}\right)$.

Step 4: Estimate $\mu_{1}^{*}=\mathbb{E}\left\{Y_{i}(1)\right\}$ by the Horvitz-Thompson estimator $\widehat{\mu}_{1}=\frac{1}{n} \sum_{i=1}^{n} T_{i} Y_{i} / \widetilde{\pi}_{i}$.

In Step 1, we obtain an initial estimate of the propensity score via the penalized M-estimation approach. We refer to the function $Q_{n}(\beta)$ as the generalized quasi-likelihood function, as its construction is similar to the quasi-likelihood function for generalized linear models (Wedderburn, 1974). To understand how the generalized quasi-likelihood function is motivated, we compute the corresponding quasi-score function,

$$
\frac{\partial Q_{n}(\beta)}{\partial \beta}=\frac{1}{n} \sum_{i=1}^{n}\left\{\frac{T_{i}}{\pi\left(\beta^{\top} X_{i}\right)}-1\right\} w_{1}\left(\beta^{\top} X_{i}\right) X_{i} .
$$

Since (2.11) is an unbiased estimating function for $\beta, Q_{n}(\beta)$ serves as a legitimate quasi-likelihood function that integrates the quasi-score function (2.11). The quasi-likelihood function $Q_{n}(\beta)$ depends on the choice of weighting function $w_{1}(u)$. In particular, we consider the following two examples. 
(a) If $w_{1}(u)=\pi(u),(2.11)$ is identical to the score function for the logistic regression and thus $Q_{n}(\beta)$ reduces to the standard quasi-likelihood function for the treatment variable.

(b) If $w_{1}(u)=1$, the quasi-score function (2.11) leads to the strong covariate balancing equation (2.4). Consequently, we call $Q_{n}(\beta)$ with $w_{1}(u)=1$ as the covariate balancing loss function.

Thus, in Step 1, we allow a broad class of initial estimators $\widehat{\beta}$, including the penalized (quasi)maximum likelihood estimator and many other penalized M-estimators corresponding to different $w_{1}(u)$. By computing the Hessian matrix of $Q_{n}(\beta)$, we find that $(2.7)$ can be a non-convex optimization problem depending on the choice of $w_{1}(u)$. The non-convexity may pose computational challenges. For instance, the gradient descent algorithm can be trapped at a local solution which is far from the global maximizer. To avoid the computational issue, we mainly focus on the concave quasi-likelihood function $Q_{n}(\beta)$. It is easy to verify that $Q_{n}(\beta)$ with $w_{1}(u)=\pi(u)$ in case (a) and $w_{1}(u)=1$ in case $(\mathrm{b})$ are both concave.

In Step 2, we fit the outcome model using a class of penalized weighted least square estimators. We allow the weight $w_{2}\left(\widehat{\beta}^{\top} X_{i}\right)$ to depend on $X_{i}$ and also the initial estimator $\widehat{\beta}$ from Step 1. For instance, we have the following examples.

(a') If $w_{2}(u)=1, L_{n}(\alpha)$ is the classical least square loss function in the treatment group.

(b') If $w_{2}(u)=1 / \pi(u), L_{n}(\alpha)$ is known as the inverse propensity score weighted least square loss.

(c') If $w_{2}(u)=\pi^{\prime}(u) / \pi^{2}(u), L_{n}(\alpha)$ remains a valid loss function for estimating $\alpha$. It is shown in Section 3.4 that this loss function plays an important role when studying the robustness of the proposed estimator to misspecified outcome models. In the following, we call this loss function as the propensity score adjusted least square loss.

Step 3 removes the bias induced by the penalized estimators used in Steps 1 and 2. We calibrate the estimated propensity score by balancing a subset of covariates $X_{\widetilde{S}}$, which represent the variables selected for the outcome model. Equation (2.10) implies that the proposed HD-CBPS methodology achieves the strong covariate balancing property only for these covariates $X_{\widetilde{S}}$ but not for the other covariates $X_{\widetilde{S}^{c}}$. Thus, unlike the original CBPS methodology, the HD-CBPS methodology does not achieve the strong covariate balancing property. Interestingly, however, the HD-CBPS methodology does approximately satisfy the weak covariate balancing property if $\alpha^{*}$ can be well approximated by $\widetilde{\alpha}$. Specifically, we have

$$
\sum_{i=1}^{n}\left(\frac{T_{i}}{\widetilde{\pi}_{i}}-1\right) \alpha^{* \top} X_{i} \approx \sum_{i=1}^{n}\left(\frac{T_{i}}{\widetilde{\pi}_{i}}-1\right) \widetilde{\alpha}^{\top} X_{i}=\sum_{i=1}^{n}\left(\frac{T_{i}}{\widetilde{\pi}_{i}}-1\right) \widetilde{\alpha}_{\widetilde{S}^{\top}}^{\top} X_{i \widetilde{S}}=0
$$

where the first equality follows from $\widetilde{\alpha}_{\widetilde{S}^{c}}=0$ and the second equality holds due to equation (2.10).

In Step 4, we estimate $\mu_{1}^{*}$ using the Horvitz-Thompson estimator. In the following, we comment on the connection between the proposed estimator and the other commonly used estimators. First, our estimator can be written as the Horvitz-Thompson estimator with the normalized weights, 
which is known as the Hajek estimator (Hájek, 1971),

$$
\widehat{\mu}_{1}=\frac{1}{n} \sum_{i=1}^{n} \frac{T_{i} Y_{i}}{\widetilde{\pi}_{i}}=\frac{\sum_{i=1}^{n} T_{i} Y_{i} / \widetilde{\pi}_{i}}{\sum_{i=1}^{n} T_{i} / \widetilde{\pi}_{i}},
$$

The second equality follows because $\sum_{i=1}^{n}\left(T_{i} / \widetilde{\pi}_{i}-1\right) / n=0$ so long as an intercept is included in $X_{i \widetilde{S}}$. Imbens et al. (2005) and Busso et al. (2014) showed that the normalized Horvitz-Thompson estimator tends to be more stable than the unnormalized version numerically. Thus, we expect that the proposed estimator has a better finite sample performance than the standard (i.e., unnormalized) Horvitz-Thompson estimator.

Second, our estimator can be also rewritten as an AIPW estimator with the linear outcome model (Robins et al., 1994),

$$
\widehat{\mu}_{1}=\frac{1}{n} \sum_{i=1}^{n} \frac{T_{i} Y_{i}}{\tilde{\pi}_{i}}=\frac{1}{n} \sum_{i=1}^{n} \frac{T_{i} Y_{i}}{\widetilde{\pi}_{i}}+\frac{1}{n} \sum_{i=1}^{n}\left(1-\frac{T_{i}}{\widetilde{\pi}_{i}}\right) \widetilde{\alpha}^{\top} X_{i}
$$

where the second equality follows from two equalities in (2.12). We conduct a further technical comparison with the AIPW estimator in Section 3.5.

From a practical perspective, Rubin (2008) advocated an outcome free design for the treatment effect estimation, in which the propensity score is estimated without reference to the outcome information in a similar spirit to the randomized experiment. We note that our estimator does not fall into the framework of the outcome free design, because we recalibrate the propensity score using the outcome information in Step 3.

Our procedure differs from the existing methods on high-dimensional regressions; see Zhang and Zhang (2014), Javanmard and Montanari (2013), van de Geer et al. (2014), Belloni et al. (2016), Ning and Liu (2017), Cai and Guo (2015), and Dukes et al. (2018), among many others. The main idea of these methods is to correct the bias of the Lasso-type estimators or the score function by inverting the optimality condition or projecting to the tangent space of the nuisance function. In contrast, we remove the bias of the Lasso estimators $\widehat{\beta}$ and $\widetilde{\alpha}$ using a covariate balancing strategy.

\section{Theoretical Properties of the Proposed Estimator}

We now study the theoretical properties of the proposed estimator. We begin by presenting the required assumptions.

\subsection{Assumptions}

Assumption 3.1 (Unconfoundedness). The treatment assignment is unconfounded, i.e., $\left\{Y_{i}(1), Y_{i}(0)\right\} \perp$ $T_{i} \mid X_{i}$.

Assumption 3.2 (Overlap). There exists a constant $c_{0}>0$ such that $\pi_{i}^{*} \geq c_{0}$ for $1 \leq i \leq n$, where $\pi_{i}^{*}=\pi\left(X_{i}^{\top} \beta^{*}\right)$ 
Assumption 3.1 implies that there is no unmeasured confounders while Assumption 3.2 requires that all samples have a positive probability to receive the treatment. Together, these represent the standard strong ignorability condition common to propensity score methods (Rosenbaum and Rubin, 1983); see e.g., Assumption 1 of Farrell (2015) and Assumption 6 of Athey et al. (2016). To estimate the treatment effect, one also needs to identify $\mathbb{E}\{Y(0)\}$, which requires a similar overlap assumption $\pi_{i}^{*} \leq 1-c_{1}$ for some constant $c_{1}>0$.

Assumption 3.3 (Sub-Gaussian condition). Assume that $\epsilon_{1}=Y(1)-\alpha^{* \top} X$ and $X_{j}$ satisfy $\left\|\epsilon_{1}\right\|_{\psi_{2}} \leq C_{\epsilon}$ and $\left\|X_{j}\right\|_{\psi_{2}} \leq C_{X}$ for any $1 \leq j \leq d$, where $C_{X}$ and $C_{\epsilon}$ are positive constants.

Assumption 3.3 controls the tail behavior of the error $\epsilon_{1}$ and the covariate $X_{j}$, which facilitates the use of many existing concentration inequalities in high-dimensional statistics. Similar subGaussian conditions are imposed by Athey et al. (2016) in their Theorem 5. Belloni et al. (2013a); Farrell (2015) relaxed the sub-Gaussian condition on $\epsilon_{1}$ to the bounded $q$ th moment for some $q>4$ under a slightly stronger sparsity assumption than our sparsity assumption below.

Assumption 3.4 (Sparsity). Assume that $\left(s_{1} \vee s_{2}\right) \log (d \vee n) / n^{1 / 2}=o(1)$ as $s_{1}, s_{2}, d, n \rightarrow \infty$, where $s_{1}=\left\|\beta^{*}\right\|_{0}$ and $s_{2}=\left\|\alpha^{*}\right\|_{0}$. Recall that $a \vee b=\max (a, b)$.

Assumption 3.4 requires that the propensity score model and outcome model are sparse. Since we consider the high-dimensional case with $d \gg n$, the sparsity assumption plays an important role in the regularized M-estimation of the propensity score model and outcome model. In particular, if $s_{1} \asymp s_{2} \asymp n^{\kappa}$ for some $\kappa<1 / 2$, then the condition reduces to $d=o\left(\exp \left(n^{1 / 2-\kappa}\right)\right)$. This condition is similar to that in Belloni et al. (2013a, 2014) and Farrell (2015), where they imposed a slightly stronger condition with $\log (d \vee n)$ replaced by $\{\log (d \vee n)\}^{q}$ for some $q>1$.

This sparsity assumption will be further relaxed later in the paper. To preview these results, we note that Remark 3.8 of Section 3.2 discusses the approximate sparsity assumption and Remark 3.9 of Section 3.2 considers a simple modification of the algorithm based on the sample splitting approach (Chernozhukov et al., 2016), which requires a weaker sparsity assumption $\left(s_{1} s_{2}\right)^{1 / 2} \log (d \vee$ $n) / n^{1 / 2}=o(1)$.

Assumption 3.5 (Eigenvalue condition). Denote $\Sigma=\mathbb{E}\left(X^{\otimes 2}\right)$. There exists a constant $C>0$ such that $C \leq \lambda_{\min }\left(\Sigma_{S S}\right) \leq \lambda_{\max }\left(\Sigma_{S S}\right) \leq 1 / C$ for any $S \subset\{1, \ldots, d\}$ with $|S| \lesssim\left(s_{1} \vee s_{2}\right) \log n$.

When the dimension $d$ is fixed, this assumption simply requires that the design matrix has full column rank, which is a standard regularity condition for regression problems. In high dimension, Assumption 3.5 implies the well known sparse eigenvalue condition introduced by Bickel et al. (2009) to study the Lasso estimator; see Lemma 1 in Belloni et al. (2013b). The same sparse eigenvalue condition is imposed by Belloni et al. (2014). We also refer to Assumption 5 of Athey et al. (2016) and Section 6.2 of Farrell (2015) for a similar restricted eigenvalue condition. Since our assumption only applies to any $S$ by $S$ submatrix of $\Sigma$, it is weaker than $C \leq \lambda_{\min }(\Sigma) \leq \lambda_{\max }(\Sigma) \leq 1 / C$, imposed by van de Geer et al. (2014), Ning and Liu (2017), and Cai and Guo (2015) for highdimensional inference.

Assumption 3.6 (Propensity score and weight functions). Assume that $Q_{n}(\beta)$ is a concave function. Let $C, C^{\prime}$ denote positive constants, which may change from line to line. 
(1) The propensity score model $\pi(u)$ satisfies $C \leq\left(\pi_{i}^{*}\right)^{\prime} \leq 1 / C$, and there exist constants $r>0$ and $C^{\prime}>0$ such that the Lipschitz condition holds locally, i.e., $\left|\pi^{\prime}(u)-\pi^{\prime}(v)\right| \leq C^{\prime}|u-v|$ for any $u, v \in\left[X_{i}^{\top} \beta^{*}-r, X_{i}^{\top} \beta^{*}+r\right]$ and $1 \leq i \leq n$.

(2) The weight $w_{1}(u)$ satisfies $C \leq w_{1 i}^{*} \leq 1 / C,\left(w_{1 i}^{*}\right)^{\prime} \leq 1 / C$, and the local Lipschitz condition $\left|w_{1}^{\prime}(u)-w_{1}^{\prime}(v)\right| \leq C^{\prime}|u-v|$ for any $u, v \in\left[X_{i}^{\top} \beta^{*}-r, X_{i}^{\top} \beta^{*}+r\right]$ and $1 \leq i \leq n$, where $w_{1 i}^{*}=w_{1}\left(X_{i}^{\top} \beta^{*}\right)$.

(3) The weight $w_{2}(u)$ satisfies $C \leq w_{2 i}^{*} \leq 1 / C$ and $\left(w_{2 i}^{*}\right)^{\prime} \leq 1 / C$ for $1 \leq i \leq n$, where $w_{2 i}^{*}=$ $w_{2}\left(X_{i}^{\top} \beta^{*}\right)$. Assume $w_{2}^{\prime}(u)$ is continuous.

Finally, Assumption 3.6 imposes mild regularity conditions on the propensity score function and weight functions. In part (1), we assume $\pi(u)$ is differentiable and its derivative is bounded and Lipschitz around $X_{i}^{\top} \beta^{*}$. Under the overlap assumption $c_{1} \leq \pi_{i}^{*} \leq 1-c_{1}$, part (1) holds for the logistic regression without any further conditions. In part (2) and (3), we assume mild conditions on the magnitude and smoothness of $w_{1}(u)$ and $w_{2}(u)$. Again, if $\pi(u)$ is the logistic function and the overlap assumption holds, all examples of $w_{1}(u)$ and $w_{2}(u)$ discussed in Section 2.2 satisfy the regularity conditions in part (2) and (3). Thus, Assumption 3.6 holds for the logistic propensity score model without any further conditions.

\subsection{Asymptotic Distribution under Correct Model Specification}

In this subsection, we derive the theoretical results for the proposed HD-CBPS estimator $\widehat{\mu}_{1}$ when both the propensity score model (2.2) and the outcome model (2.3) are correctly specified. Recall that our estimator $\widehat{\mu}_{1}$ depends on the choice of the two weight functions, i.e., $w_{1}(u)$ in Step 1 and $w_{2}(u)$ in Step 2. In the following, we establish the asymptotic normality and semiparametric efficiency of $\widehat{\mu}_{1}$ for any weight function $w_{1}(u)$ and $w_{2}(u)$.

Theorem 3.7 (Asymptotic Normality and Semiparametric Efficiency). Suppose that both the propensity score model (2.2) and the outcome model (2.3) are correctly specified and Assumptions 1-6 hold. If we take $\lambda \asymp \lambda^{\prime} \asymp\{\log (d \vee n) / n\}^{1 / 2}$, then the estimator $\widehat{\mu}_{1}$ with any weight function $w_{1}(u)$ and $w_{2}(u)$ satisfies

$$
\widehat{\mu}_{1}-\mu_{1}^{*}=\frac{1}{n} \sum_{i=1}^{n}\left[\frac{T_{i}}{\pi_{i}^{*}}\left\{Y_{i}(1)-\alpha^{* \top} X_{i}\right\}+\alpha^{* \top} X_{i}-\mu_{1}^{*}\right]+O_{p}\left(\frac{\left(s_{1} \vee s_{2}\right) \log (d \vee n)}{n}\right),
$$

as $s_{1}, s_{2}, d, n \rightarrow \infty$. Let $V$ be the semiparametric asymptotic variance bound, i.e.,

$$
V=\mathbb{E}\left\{\frac{1}{\pi^{*}} \mathbb{E}\left(\epsilon_{1}^{2} \mid X\right)+\left(\alpha^{* \top} X-\mu_{1}^{*}\right)^{2}\right\}
$$

Assume that $\mathbb{E}\left(\epsilon_{1}^{2} \mid X\right) \geq c$ for some constant $c>0$ and $\mathbb{E}\left(\alpha^{* \top} X\right)^{4}=O\left(s_{2}^{2}\right)$. Then, $n^{1 / 2}\left(\widehat{\mu}_{1}-\right.$ $\left.\mu_{1}^{*}\right) / V^{1 / 2} \rightarrow_{d} N(0,1)$.

The theorem shows that $\widehat{\mu}_{1}-\mu_{1}^{*}$ is asymptotically equivalent to the average of the efficient score functions and hence $\widehat{\mu}_{1}$ is locally efficient under the correct model specification. In addition, the 
asymptotic distribution of $\widehat{\mu}_{1}$ does not depend on choice of the weight functions $w_{1}(u)$ and $w_{2}(u)$, provided that they satisfy Assumption 3.6. The intuition is that, as long as the weak covariate balancing property is approximately attained, the choice of the weight functions in the first two steps is less important.

To prove the asymptotic normality of $\widehat{\mu}_{1}$, we further require that the variance of the noise cannot tend to 0 , i.e., $\mathbb{E}\left(\epsilon_{1}^{2} \mid X\right) \geq c>0$. This guarantees the non-degeneracy of the asymptotic variance $V$. We also assume $\mathbb{E}\left(\alpha^{* \top} X\right)^{4}=O\left(s_{2}^{2}\right)$ in order to verify the Lyaponov condition for the central limit theorem. This is a mild technical condition. For instance, if $X$ is a sub-Gaussian vector and $\left\|\alpha^{*}\right\|_{2}=O\left(s_{2}^{1 / 2}\right)$, then $\left\|\alpha^{* \top} X\right\|_{\psi_{2}} \leq\left\|\alpha^{*}\right\|_{2}\|X\|_{\psi_{2}}=O\left(s_{2}^{1 / 2}\right)$. This further implies the desired condition $\mathbb{E}\left(\alpha^{* \top} X\right)^{4}=O\left(s_{2}^{2}\right)$ by the definition of the sub-Gaussian norm.

We note that the asymptotic variance $V$ depends on the true data generating process, which is allowed to change with $d$ and also $n$. For this reason, we consider the limiting distribution of the standardized statistic $n^{1 / 2}\left(\widehat{\mu}_{1}-\mu_{1}^{*}\right) / V^{1 / 2}$ as $n, d \rightarrow \infty$. Hahn (1998) proved that $V$ is the semiparametric asymptotic variance bound, when both the propensity score and outcome models are treated as nuisance. He further proposed a nonparametric IPW estimator for fixed $d$ that attains this semiparametric efficiency bound. We show that, when the high-dimensional models (2.2) and (2.3) are both correctly specified, the estimator $\widehat{\mu}_{1}$ attains the same bound and hence locally efficient.

Our variance bound $V$ is different from the "oracle efficiency bound", which is the semiparametric variance bound with the known support of the propensity score and outcome models (Hahn, 2004). Since the support of both models is unknown and the variable selection consistency does not hold under our assumptions, the estimation of the support set leads to additional uncertainty. This explains why our method cannot attain the oracle efficiency bound. We refer to Section 5.3 of Farrell (2015) for further discussion on this point.

Since our goal is to estimate the causal effects rather than the coefficients in the propensity score and outcome models, we show that the asymptotic normality of $\widehat{\mu}_{1}$ does not rely on the variable selection consistency in either model. It is known that variable selection consistency requires more stringent conditions, e.g., signal strength condition and irrepresentable condition (Zhao and Yu, 2006). Theorem 3.7 does not require these conditions.

Remark 3.8 (Approximate Sparsity). Theorem 3.7 assumes that the propensity score and outcome models are sparse. However, the same conclusion holds for the class of approximately sparse models. Specifically, assume that

$$
\mathbb{E}\left(Y_{i}(1) \mid X_{i}\right)=X_{i}^{\top} \alpha^{*}+r_{i}, \text { and } \mathbb{P}\left(T_{i}=1 \mid X_{i}\right)=\pi\left(X_{i}^{\top} \beta^{*}+u_{i}\right)
$$

where $s_{1}=\left\|\beta^{*}\right\|_{0}$ and $s_{2}=\left\|\alpha^{*}\right\|_{0}$ and $r_{i}, u_{i}$ are the approximation errors. By introducing $r_{i}, u_{i}$ in these models, we allow for the nonlinear effect of $X_{i}$ and the non-sparse effect due to weak signals in the models. Using a proof similar to the one for the theorem, we can show that Theorem 3.7 holds so long as the approximation errors satisfy

$$
\sum_{i=1}^{n} r_{i}^{2}=O\left(s_{2}\right), \sum_{i=1}^{n} u_{i}^{2}=O\left(s_{1}\right) \text { and } \sum_{i=1}^{n} r_{i} u_{i}=o\left(n^{1 / 2}\right) .
$$


Thus, our results are robust to the minor violations of the linearity and sparsity assumptions.

Remark 3.9 (Sample Splitting). In a recent work, Chernozhukov et al. (2016) proposed a double machine learning method based on the sample splitting technique to relax the sparsity assumption. In the supplementary materials, we proposed a modified algorithm based on the sample splitting, so that Assumption 3.4 is relaxed to a weaker assumption $\left(s_{1} s_{2}\right)^{1 / 2} \log (d \vee n) / n^{1 / 2}=o(1)$. Ignoring the logarithmic factors of $d$ and $n$, Assumption 3.4 requires $s_{1}=o\left(n^{1 / 2}\right)$ and $s_{2}=o\left(n^{1 / 2}\right)$. In contrast, by using the sample splitting technique, we only require a weaker condition $s_{1} s_{2}=o(n)$, which may still hold if one model is dense (e.g., $n^{1 / 2} \ll s_{1} \ll n$ ) and the other model is sufficiently sparse (e.g., $s_{2} \ll n^{1 / 2}$ ). However, the sample splitting method incurs further computational cost and may not be stable when the sample size is relatively small.

Remark 3.10 (Sample Boundedness). Unlike many of the existing estimators, the proposed HDCBPS method guarantees that $\widehat{\mu}_{1}$ lies in the range of $\left\{Y_{i}: T_{i}=1, i=1, \ldots, n\right\}$. This sample boundedness property (Robins et al., 2007) holds because, by construction, the covariate balancing equation satisfies

$$
\frac{1}{n} \sum_{i=1}^{n}\left(\frac{T_{i}}{\widetilde{\pi}_{i}}-1\right)=0
$$

so long as an intercept is included in $X_{i \widetilde{S}}$. Equation (3.1) implies that the estimated propensity score $\widetilde{\pi}_{i}$ must be greater than or equal to $1 / n$ for any treated observation. In contrast, the estimated propensity score $\pi_{i}^{*}$ for the $i$ th observation via the penalized maximum likelihood estimation can become very close to 0 , leading to extremely large weights for some observations and unstable causal effect estimates. To see why the sample boundedness property holds, we have

$$
\frac{1}{n} \sum_{i=1}^{n} \frac{T_{i} Y_{i}}{\widetilde{\pi}_{i}} \geq \frac{\min _{i: T_{i}=1} Y_{i}}{n} \sum_{i=1}^{n} \frac{T_{i}}{\widetilde{\pi}_{i}}=\min _{i: T_{i}=1} Y_{i},
$$

where the last equality follows from equation (3.1). Similarly, we can show that $\widehat{\mu}_{1} \leq \max _{i: T_{i}=1} Y_{i}$.

Finally, to construct a confidence interval for $\mu_{1}^{*}$, we estimate $V$ by

$$
\widehat{V}=\frac{1}{n} \sum_{i=1}^{n}\left\{\frac{T_{i}}{\tilde{\pi}_{i}^{2}}\left(Y_{i}-\widetilde{\alpha}^{\top} X_{i}\right)^{2}+\left(\widetilde{\alpha}^{\top} X_{i}-\widehat{\mu}_{1}\right)^{2}\right\} .
$$

The following corollary shows that $\widehat{V}$ is a consistent estimator of $V$ and therefore we obtain valid confidence intervals for $\mu_{1}^{*}$.

Corollary 3.11 (Honest Confidence Intervals). Suppose that the assumptions in Theorem 3.7 hold. Then,

$$
|\widehat{V}-V|=O_{p}\left(\left(s_{1} \vee s_{2}\right) \sqrt{\frac{\log (d \vee n)}{n}}\right) .
$$

Given $0<\eta \leq 1$, define the $(1-\eta)$-confidence interval as $\mathcal{I}=\left(\widehat{\mu}_{1}-z_{1-\eta / 2}(\widehat{V} / n)^{1 / 2}, \widehat{\mu}_{1}+\right.$ 
$\left.z_{1-\eta / 2}(\widehat{V} / n)^{1 / 2}\right)$, where $z_{1-\eta / 2}$ is the $(1-\eta / 2)$ quantile of a standard normal distribution. Then,

$$
\left|\mathbb{P}\left(\mu_{1}^{*} \in \mathcal{I}\right)-(1-\eta)\right|=o(1) .
$$

Indeed, this confidence interval $\mathcal{I}$ is honest in the sense that equation (3.3) holds uniformly over all probability distributions that satisfy Assumptions 1-6. In addition, the proof of Corollary 3.11 holds even if the error $\epsilon_{1}$ is heteroskedastic, i.e., $\mathbb{E}\left(\epsilon_{1}^{2} \mid X\right)$ depends on the value of $X$.

In the Supplementary Materials, we further extend these theoretical results to the estimation of the average treatment effect for the treated (ATT).

\subsection{Asymptotic Distribution under Misspecified Propensity Score Models}

We next investigate the robustness of the proposed HD-CBPS methodology to the misspecification of propensity score model. In this subsection, we assume that the true propensity score $\pi^{*}=\mathbb{P}(T=$ $1 \mid X)$ does not belong to the assumed parametric class $\left\{\pi\left(X^{\top} \beta\right): \beta \in \mathbb{R}^{d}\right\}$. To study the limiting behavior of our estimator $\widehat{\mu}_{1}$ in this setting, we first define the estimand of $\widehat{\beta}$ in Step 1 . Given the generalized quasi-likelihood function $Q_{n}(\beta)$, the estimand of $\widehat{\beta}$ in $(2.7)$ is defined as

$$
\beta^{o}=\operatorname{argmax} \mathbb{E}\left[\int_{0}^{\beta^{\top} X_{i}}\left\{\frac{T_{i}}{\pi(u)}-1\right\} w_{1}(u) d u\right] .
$$

We note that the estimand $\beta^{o}$ implicitly depends on the choice of the weight function $w_{1}(u)$, and when the model is correctly specified, $\beta^{o}$ reduces to $\beta^{*}$. In the following proposition, we assume that the estimand $\beta^{o}$ is sparse, which is a technical assumption required to study misspecified models in high-dimensional settings (Bühlmann and van de Geer, 2015). For instance, under this assumption, it can be shown that $\beta^{o}$ can be consistently estimated by $\widehat{\beta}$. Without similar assumptions, the high-dimensional parameter $\beta^{o}$ may not be estimable. The following proposition establishes the asymptotic properties of $\widehat{\mu}_{1}$ under misspecified propensity score models.

Proposition 3.12. (Consistency and Asymptotic Normality under Misspecified PropenSITY Score Models) Suppose that the outcome model (2.3) is correctly specified, but the propensity score model (2.2) is misspecified. Assumptions 1-6 hold with $\beta^{*}$ replaced by $\beta^{o}$. If we take $\lambda \asymp \lambda^{\prime} \asymp\{\log (d \vee n) / n\}^{1 / 2}$, then the estimator $\widehat{\mu}_{1}$ with any weight functions $w_{1}(u)$ and $w_{2}(u)$ satisfies

$$
\widehat{\mu}_{1}-\mu_{1}^{*}=O_{p}\left(\sqrt{\frac{\left(s_{1} \vee s_{2}\right) \log (d \vee n)}{n}}\right) .
$$

Moreover, if we set $w_{1}(u)=1$, then for any $w_{2}(u)$ we have

$$
\widehat{\mu}_{1}-\mu_{1}^{*}=\frac{1}{n} \sum_{i=1}^{n}\left\{\frac{T_{i}}{\pi_{i}^{o}}\left(Y_{i}(1)-\alpha^{* \top} X_{i}\right)+\alpha^{* \top} X_{i}-\mu_{1}^{*}\right\}+O_{p}\left(\frac{\left(s_{1} \vee s_{2}\right) \log (d \vee n)}{n}\right)
$$


where $\pi_{i}^{o}=\pi\left(X_{i}^{\top} \beta^{o}\right)$. Assume that $\mathbb{E}\left(\epsilon_{1}^{2} \mid X\right) \geq c$ for some constant $c>0$ and $\mathbb{E}\left(\alpha^{* \top} X\right)^{4}=O\left(s_{2}^{2}\right)$. This implies $n^{1 / 2}\left(\widehat{\mu}_{1}-\mu_{1}^{*}\right) / V_{\text {mis-ps }}^{1 / 2} \rightarrow_{d} N(0,1)$ where

$$
V_{\text {mis-ps }}=\mathbb{E}\left\{\frac{\pi^{*}}{\pi^{o 2}} \mathbb{E}\left(\epsilon_{1}^{2} \mid X\right)+\left(\alpha^{* \top} X-\mu_{1}^{*}\right)^{2}\right\} .
$$

This proposition shows that our estimator $\widehat{\mu}_{1}$ remains consistent, but the convergence rate in (3.4) can be slower than $n^{-1 / 2}$. When the dimension $d$ is fixed, (3.4) reduces to $\widehat{\mu}_{1}-\mu_{1}^{*}=$ $O_{p}\left((\log n / n)^{1 / 2}\right)$, which agrees with the convergence rate of the doubly robust estimators in low dimension up to a $\log n$ factor, see Robins et al. (1994); Bang and Robins (2005); Robins et al. (2007); Cao et al. (2009); Tan (2010); Van der Laan and Rose (2011); Vermeulen and Vansteelandt (2015). However, in high dimension, the convergence rate becomes slower because of the extra bias in the propensity score estimation when the model is misspecified.

The second part of this proposition states that, if we carefully choose the weight function $w_{1}(u)=1$ in the algorithm, the root- $n$ consistency and asymptotic normality of $\widehat{\mu}_{1}$ are restored without paying any price. Recall that with $w_{1}(u)=1$, the quasi-score function (2.11) reduces to the strong covariate balancing equation (2.4) as in the example (b). This is the key ingredient to remove the extra bias term in the asymptotic expansion of $\widehat{\mu}_{1}$ when the propensity score model is misspecified.

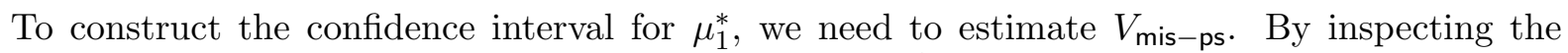
proof of Corollary 3.11, we can show that the estimator $\widehat{V}$ defined in (3.2) is still consistent for $V_{\text {mis-ps }}$, even if the propensity score model is misspecified. Thus, the confidence interval shown in Corollary 3.11 is valid whether or not the propensity score model is misspecified. Finally, we note that if the propensity score model is correctly specified, i.e., $\pi_{i}^{o}=\pi_{i}^{*}$, then the asymptotic variance $V_{\text {mis-ps }}$ in Proposition 3.12 reduces to the asymptotic variance $V$ in Theorem 3.7.

\subsection{Asymptotic Distribution under Misspecified Outcome Models}

In this subsection, we study the robustness of the proposed HD-CBPS methodology to the misspecification of the outcome model. Assume that the propensity score model is correct, but the true conditional mean function $\mathbb{E}\left(Y_{i}(1) \mid X_{i}=x\right)$ is nonlinear in $x$, i.e., there does not exist $\alpha^{*}$ such that $\mathbb{E}\left(Y_{i}(1) \mid X_{i}=x\right)=\alpha^{* \top} x$. Similar to Section 3.3, we define the estimand of $\widetilde{\alpha}$ in Step 2 as

$$
\alpha^{o}=\operatorname{argmin} \mathbb{E}\left\{T_{i} w_{2}\left(\beta^{* \top} X_{i}\right)\left(Y_{i}-\alpha^{\top} X_{i}\right)^{2}\right\}
$$

which in turn depends on the weight function $w_{2}\left(\beta^{* \top} X_{i}\right)$. The following proposition establishes the asymptotic properties of $\widehat{\mu}_{1}$ under misspecified outcome models.

Proposition 3.13. (Consistency and Asymptotic Normality under Misspecified OutCome Models) Suppose that the propensity score model (2.2) is correctly specified, but the outcome model (2.3) is misspecified. Assumptions 1-6 hold with $\alpha^{*}$ replaced by $\alpha^{o}$. If we take $\lambda \asymp \lambda^{\prime} \asymp\{\log (d \vee n) / n\}^{1 / 2}$, then the estimator $\widehat{\mu}_{1}$ with any weight functions $w_{1}(u)$ and $w_{2}(u)$ 
satisfies

$$
\widehat{\mu}_{1}-\mu_{1}^{*}=O_{p}\left(\sqrt{\frac{\left(s_{1} \vee s_{2}\right) \log (d \vee n)}{n}}\right) .
$$

Moreover, if we set $w_{2}(u)=\pi^{\prime}(u) / \pi^{2}(u)$, then for any $w_{1}(u)$ we have

$$
\widehat{\mu}_{1}-\mu_{1}^{*}=\frac{1}{n} \sum_{i=1}^{n}\left\{\frac{T_{i}}{\pi_{i}^{*}}\left(Y_{i}(1)-\alpha^{o \top} X_{i}\right)+\alpha^{o \top} X_{i}-\mu_{1}^{*}\right\}+O_{p}\left(\frac{\left(s_{1} \vee s_{2}\right) \log (d \vee n)}{n}\right) .
$$

Assume that $\mathbb{E}\left(\epsilon_{1}^{o 2} \mid X\right) \geq c$ for some constant $c>0$ and $\mathbb{E}\left(\alpha^{o \top} X\right)^{4}=O\left(s_{2}^{2}\right)$, where $\epsilon_{1}^{o}=Y(1)-$ $\alpha^{o \top} X$. This implies $n^{1 / 2}\left(\widehat{\mu}_{1}-\mu_{1}^{*}\right) / V_{\text {mis }-\mathrm{o}}^{1 / 2} \rightarrow_{d} N(0,1)$, where

$$
V_{\text {mis }-\circ}=\mathbb{E}\left\{\frac{1}{\pi^{*}} \mathbb{E}\left(\epsilon^{o 2} \mid X\right)+\left(\alpha^{o \top} X-\mu_{1}^{*}\right)^{2}\right\} .
$$

The results in this proposition are parallel to those in Proposition 3.12. Specifically, when the outcome model is misspecified, our estimator is still consistent, but has a slower convergence rate as shown in (3.5). However, as long as we choose $w_{2}(u)=\pi^{\prime}(u) / \pi^{2}(u)$ or equivalently the propensity score adjusted least square loss $L_{n}(\alpha)$ in example (c') of Section 2.2, the desired properties such as the root- $n$ consistency and asymptotic normality are restored. The form of the $w_{2}(u)$ is designed to eliminate the bias term in the expansion of $\widehat{\mu}_{1}$ under the misspecified outcome model. Finally, the estimator $\widehat{V}$ in (3.2) is consistent for the asymptotic variance $V_{\text {mis-o }}$.

Remark 3.14 (Double Robustness and Honest Confidence Intervals). Propositions 3.12 and 3.13 together imply that our estimator $\widehat{\mu}_{1}$ is root- $n$ consistent and asymptotically normal provided either the propensity score model or outcome model is correctly specified. This estimator does not require to know which of the two models is correct. Since $\widehat{V}$ is always consistent, the same confidence interval defined in Corollary 3.11 is valid as long as one of the two models is correctly specified. Thus, we recommend the use of this estimator and the associated confidence interval in practice. Finally, we comment that when $w_{1}(u)=1$ and $w_{2}(u)=\pi^{\prime}(u) / \pi^{2}(u)$, the gradients of $Q_{n}(\beta)$ and $L_{n}(\alpha)$ reduce to the estimating equations proposed by Robins et al. (2007).

\subsection{Comparison with the Related Work}

In this subsection, we compare our method with the related work. First, we comment on the theoretical results of the AIPW estimator (Belloni et al., 2013a; Farrell, 2015) and double selection estimator (Belloni et al., 2014) when both the propensity score and outcome models are correctly specified. Second, we compare the results when one of the two models is misspecified. Finally, we consider the more recent work by Athey et al. (2016), Zhao (2016) and Tan (2017, 2018).

When both the propensity score and outcome models are correctly specified, Belloni et al. (2013a) and Farrell (2015) showed that the AIPW estimator is asymptotically normal and efficient in high dimension. Their assumptions and main results are parallel to our Theorem 3.7. However, the sample boundedness property in Remark 3.10 does not hold for the AIPW estimator in general. We note that the above work and our Theorem 3.7 can be viewed as an extension of the semiparametric 
efficiency property of the doubly robust estimators; see Robins et al. (1994); Bang and Robins (2005); Robins et al. (2007); Cao et al. (2009); Tan (2010); Van der Laan and Rose (2011); Vermeulen and Vansteelandt (2015), among many others.

When either the propensity score model or the outcome model is misspecified, Propositions 3.12 and 3.13 provide a complete characterization of the asymptotic behavior of our estimator. In the same context, Farrell (2015) proved that the AIPW estimator is consistent, but Theorem 2 of that work does not yield an explicit convergence rate. In fact, we show in the supplementary material that the AIPW estimator has the same convergence rate as in (3.4), which is slower than $n^{-1 / 2}$, and thus the confidence intervals for the treatment effect are not available under model misspecification. In contrast, our estimator is root- $n$ consistent, which leads to honest confidence intervals as shown in Sections 3.3 and 3.4. Indeed, this robustness of the asymptotic distributions to model specification is the main advantage over the AIPW estimators (Belloni et al., 2013a; Farrell, 2015) and the double selection estimator (Belloni et al., 2014).

Unlike our work, the approximate residual balancing method proposed by Athey et al. (2016) does not require the propensity score model to be sparse or even well formulated. Thus, their method is robust to the misspecification of the propensity score model under the assumption that the outcome model is correct. In contrast, our method requires both models to be sparse. The advantage of our framework is that it tolerates the misspecified outcome model, so long as the propensity score model is correctly specified. Thus, our work and Athey et al. (2016) are complementary to one another. In addition, when the propensity score model is correctly specified and is indeed sparse, the estimation of the propensity score can help scientists better understand the treatment assignment mechanism (e.g., Rubin, 2008). Recall that $\widetilde{\pi}_{i}=\pi\left(\widetilde{\beta}^{\top} X_{i}\right)$. As a byproduct of Theorem 3.7, our estimated propensity score is uniformly consistent,

$$
\max _{1 \leq i \leq n}\left|\widetilde{\pi}_{i}-\pi_{i}^{*}\right|=O_{p}\left\{\frac{\left(s_{1} \vee s_{2}\right) \log (d \vee n)(\log n)^{1 / 2}}{n^{1 / 2}}\right\} .
$$

Thus, the estimated propensity score $\widetilde{\pi}_{i}$ is an accurate approximation to the unknown treatment assignment mechanism. In contrast, the approximate residual balancing method does not yield an estimate of the propensity score. Finally, we note that the linearity assumption of the outcome model plays an important role in Athey et al. (2016) and their approximate residual balancing method is not readily applicable if the outcome model is nonlinear. In contrast, our method is robust to the misspecification of the outcome model and also can be generalized to nonlinear outcome models. As an illustration of its generalizability, we consider the extension of the proposed methodology to the generalized linear models in Section 4.

In another recent work, Zhao (2016) proposed a generalized covariate balancing method based on a class of scoring rules. Many existing covariate balancing estimators can be treated as the primal or dual problems of their optimization problem. Zhao (2016) studied the robustness of these estimators to misspecified propensity score models under the constant treatment effect model $\mathbb{E}\{Y(1)-Y(0) \mid X\}=\tau^{*}$ for some constant $\tau^{*}$. In contrast, our methodology allows for the heterogeneity of causal effects. In addition, while our work mainly focuses on the high-dimensional settings, Zhao (2016) does not provide statistical guarantees in such settings. 
Most recently, Tan $(2017,2018)$ proposed a penalized calibrated propensity score method and studied its robustness to model misspecification. Our work is closely related to Tan (2017), which can be seen as equivalent to directly plugging the initial estimator $\widehat{\beta}$ into the Horvitz-Thompson estimator with $w_{1}(u)=1$. However, this method does not balance the covariates as we did in Step 3. Corollary 3 of Tan (2017) implies that the estimator has the rate of the convergence $O_{p}\left(\left(s_{1} \log d / n\right)^{1 / 2}\right)$, which is slower than that of our estimator. In our proof, one can treat $\sum_{i=1}^{n}\left(T_{i} / \widehat{\pi}_{i}-1\right) \alpha^{* \top} X_{i}$ as the "bias" of the Horvitz-Thompson estimator, which is eliminated by the covariate balancing step, whereas this term remains in Tan (2017). In the followup paper, Tan (2018) removed this bias by constructing an AIPW estimator so that the resulting estimator is robust to model misspecification. However, our result is more general than that of Tan (2018). First, our Theorem 3.7, and Propositions 3.12 and 3.13 show that there exists a large class of estimators that is asymptotically normal under possible model misspecification. Second, our theory holds for generalized linear models as shown in Theorem 4.4, whereas Tan (2018)'s method is invalid if the propensity score model is misspecified.

\section{Covariate Balancing for Generalized Linear Models}

\subsection{Method}

In this section, we extend our method to the setting in which the outcome follows a generalized linear model. The validity of many existing methods such as those proposed by Imai and Ratkovic (2014), Fan et al. (2016), and Athey et al. (2016) critically rely on the assumption that the outcome follows a linear model with covariates $X_{i}$ or some transformations (e.g., spline basis) of $X_{i}$. Thus, generalizing the HD-CBPS to non-linear models is an important extension.

Assume that the working model for $Y_{i}(1)$ given $X_{i}$ belongs to the exponential family,

$$
p(y \mid X)=h(y, \phi) \exp \left[\frac{1}{a(\phi)}\left\{y \alpha^{* \top} X-b\left(\alpha^{* \top} X\right)\right\}\right]
$$

where $h(\cdot, \cdot), a(\cdot)$ and $b(\cdot)$ are known functions, $\phi$ is the dispersion parameter, and $\alpha^{*}$ is a $d$ dimensional vector of unknown regression parameters. For simplicity, we assume that the dispersion parameter $\phi$ is known. Given this setup, we propose the following modification of the HD-CBPS methodology described in Section 2.2.

Step 1: Fit the outcome model via the penalized maximum likelihood method within the treatment group,

$$
\widehat{\alpha}=\underset{\alpha \in \mathbb{R}^{d}}{\operatorname{argmin}}\left[-\frac{1}{n} \sum_{i=1}^{n} \frac{T_{i}}{a(\phi)}\left\{Y_{i} \alpha^{\top} X_{i}-b\left(\alpha^{\top} X_{i}\right)\right\}+\lambda_{0}\|\alpha\|_{1}\right]
$$

where $\lambda_{0}>0$ is a tuning parameter.

Step 2: This step is identical to Step 1 in Section 2.2, where the weight function $w_{1}(u)$ is replaced by $w_{1}\left(\widehat{\alpha}^{\top} X_{i}, u\right)$. This defines the initial estimator $\widehat{\beta}$. 
Step 3: Re-estimate the outcome model via the penalized weighted maximum likelihood method within the treatment group,

$$
\widetilde{\alpha}=\underset{\alpha \in \mathbb{R}^{d}}{\operatorname{argmin}}\left[-\frac{1}{n} \sum_{i=1}^{n} \frac{T_{i} w_{2}\left(\widehat{\beta}^{\top} X_{i}\right)}{a(\phi)}\left\{Y_{i} \alpha^{\top} X_{i}-b\left(\alpha^{\top} X_{i}\right)\right\}+\lambda^{\prime}\|\alpha\|_{1}\right]
$$

where $\lambda^{\prime}>0$ is a tuning parameter and $w_{2}(\cdot)$ is the weight function similar to Step 2 in Section 2.2 .

Step 4: Define $\widetilde{S}=\left\{j:\left|\widetilde{\alpha}_{j}\right|>0\right\}$ and $f(X)=b^{\prime \prime}\left(\widetilde{\alpha}^{\top} X\right) X_{\widetilde{S}}$. Compute,

$$
\widetilde{\gamma}=\underset{\gamma \in \mathbb{R}^{\mid \widetilde{S}} \mid}{\operatorname{argmin}}\left\|g_{n}(\gamma)\right\|_{2}^{2} \text { where } g_{n}(\gamma)=\frac{1}{n} \sum_{i=1}^{n}\left\{\frac{T_{i}}{\pi\left(\gamma^{\top} \bar{X}_{i \widetilde{S}}+\widehat{\beta}_{\widetilde{S}^{c}}^{\top} X_{i \widetilde{S}^{c}}\right)}-1\right\} f\left(X_{i}\right) \text {. }
$$

Set $\widetilde{\beta}=\left(\widetilde{\gamma}, \widehat{\beta}_{\widetilde{S}^{c}}\right)$ and $\widetilde{\pi}_{i}=\pi\left(\widetilde{\beta}^{\top} X_{i}\right)$.

Step 5: Estimate $\mu_{1}^{*}$ by $\widehat{\mu}_{1}=\frac{1}{n} \sum_{i=1}^{n} T_{i} Y_{i} / \widetilde{\pi}_{i}-\frac{1}{n} \sum_{i=1}^{n}\left(T_{i} / \widetilde{\pi}_{i}-1\right) b^{\prime}\left(\widetilde{\alpha}^{\top} X_{i}\right)$.

The current algorithm differs from that in Section 2.2 in the following three ways. First, Step 1 yields an initial estimator of $\alpha$, which is then incorporated into the weight function $w_{1}\left(\widehat{\alpha}^{\top} X_{i}, u\right)$. As shown later in Theorem 4.4, the choice of the weight function $w_{1}\left(\widehat{\alpha}^{\top} X_{i}, u\right)$ becomes critical when analyzing the asymptotic distribution of $\widehat{\mu}_{1}$ under misspecified propensity score models.

Second, Step 4 balances the weighted covariates $f(X)=b^{\prime \prime}\left(\widetilde{\alpha}^{\top} X\right) X_{\widetilde{S}}$ instead of $X_{i \widetilde{S}}$ as done in equation (2.10). The reason is that to achieve a similar weak covariate balancing property, one must balance a vector of functions $f(X)$ such that $b^{\prime}\left(\alpha^{* \top} X\right) \in \operatorname{span}\{f(X)\}$ where $\operatorname{span}\{f(X)\}$ represents the linear space generated by the basis functions $f(X)$. Let $S$ denote the support set for $\alpha$, i.e., $S=\left\{j:\left|\alpha_{j}^{*}\right|>0\right\}$. Since $b^{\prime}\left(\alpha^{* \top} X\right)$ is unknown in practice, we approximate $b^{\prime}\left(\alpha^{* \top} X\right)=b^{\prime}\left(\alpha_{S}^{* \top} X_{S}\right)$ by a local linear estimator $b^{\prime}\left(\widetilde{\alpha}^{\top} X\right)+b^{\prime \prime}\left(\widetilde{\alpha}^{\top} X\right)\left(\alpha^{*}-\widetilde{\alpha}\right)_{S} X_{S}$. Furthermore, if we replace $S$ by an estimator $\widetilde{S}$, this leads to the weighted covariates $f(X)=b^{\prime \prime}\left(\widetilde{\alpha}^{\top} X\right) X_{\widetilde{S}}$ in Step 4. Unfortunately, balancing $f(X)$ alone does not attain the (approximate) weak covariate balancing property, because the leading term $b^{\prime}\left(\widetilde{\alpha}^{\top} X\right)$ in the local linear approximation has not been considered. It is possible to add $b^{\prime}\left(\widetilde{\alpha}^{\top} X\right)$ into the covariate balancing equation, which leads to $f(X)=\left\{b^{\prime}\left(\widetilde{\alpha}^{\top} X\right), b^{\prime \prime}\left(\widetilde{\alpha}^{\top} X\right) X_{\widetilde{S}}\right\}$. However, doing so leads to additional technical assumptions on the eigenvalues of $f(X)$. To avoid such assumptions, we only attain "partial" covariate balancing in Step 4 by choosing $f(X)=b^{\prime \prime}\left(\widetilde{\alpha}^{\top} X\right) X_{\widetilde{S}}$.

Third, Step 5 applies the AIPW estimator rather than the Horvitz-Thompson estimator used in Section 2.2. The additional term, i.e., $\frac{1}{n} \sum_{i=1}^{n}\left(T_{i} / \widetilde{\pi}_{i}-1\right) b^{\prime}\left(\widetilde{\alpha}^{\top} X_{i}\right)$, comes from the bias due to the imbalance of $b^{\prime}\left(\widetilde{\alpha}^{\top} X\right)$. By equation (2.13), the AIPW estimator agrees with the Horvitz-Thompson estimator when the outcome model is linear. In this case, we have $b^{\prime \prime}(u)=1$ and $b^{\prime}(u)=u$ and balancing $b^{\prime \prime}\left(\widetilde{\alpha}^{\top} X\right) X_{\widetilde{S}}=X_{\widetilde{S}}$ is sufficient to remove the imbalance effect of $b^{\prime}\left(\widetilde{\alpha}^{\top} X\right)=\widetilde{\alpha}^{\top} X$. Thus, as expected, the current algorithm reduces to the one in Section 2.2 under the linear outcome model. In addition, if the outcome model is the Poisson regression, we can also apply the HorvitzThompson estimator because under this model $b^{\prime \prime}(u)=b^{\prime}(u)=\exp (u)$ and therefore balancing 
$b^{\prime \prime}\left(\widetilde{\alpha}^{\top} X\right) X_{\widetilde{S}}$ is sufficient, provided the intercept term is included.

\subsection{Theoretical Results}

Assumption 4.1 (Sub-Exponential condition). Assume that $\epsilon_{1}=Y(1)-b^{\prime}\left(\alpha^{* \top} X\right)$ satisfies $\left\|\epsilon_{1}\right\|_{\psi_{1}} \leq$ $C$ and $\max _{1 \leq i \leq n, 1 \leq j \leq d}\left|X_{i j}\right| \leq C_{n}$, where $C$ is a positive constant and we allow $C_{n}$ to increase with $n$.

Assumption 4.2 (Sparsity). Let us denote $s_{1}=\left\|\beta^{*}\right\|_{0}$ and $s_{2}=\left\|\alpha^{*}\right\|_{0}$. Assume that $C_{n}^{2}\left(s_{1} \vee\right.$ $\left.s_{2}\right) \log (d \vee n) / n^{1 / 2}=o(1)$, where $C_{n}$ is defined in Assumption 4.1.

Assumption 4.3 (Propensity score, outcome model and weight functions). Assume that $Q_{n}(\beta)$ is a concave function. Let $C, C^{\prime}$ denote positive constants, which may change from line to line.

(1) The same condition (1) in Assumption 3.6 holds for the propensity score model $\pi(u)$.

(2) In the outcome model, $b(u)$ is third order continuously differentiable and $\left|X_{i}^{\top} \alpha^{*}\right| \leq C^{\prime}$.

(3) The weight function $w_{1}(u, v)$ satisfies the following conditions in a small neighborhood of $u^{*}=X_{i}^{\top} \alpha^{*}$ and $v^{*}=X_{i}^{\top} \beta^{*}: C \leq w_{1}(u, v) \leq 1 / C, 0 \leq w_{1}^{\prime}(u, v) \leq 1 / C$, and the Lipschitz condition in $u,\left|w_{1}\left(u, v^{*}\right)-w_{1}\left(u^{\prime}, v^{*}\right)\right| \leq C^{\prime}\left|u-u^{\prime}\right|$, where $u \in\left[u^{*}-r, u^{*}+r\right], v \in\left[v^{*}-r, v^{*}+r\right]$ for some small constant $r>0$ and $w_{1}^{\prime}(u, v)=\partial w_{1}(u, v) / \partial v$.

(4) The same condition (3) in Assumption 3.6 holds for the weight $w_{2}(u)$.

Unlike the sub-Gaussian condition in Assumption 3.3, we allow the error $\epsilon_{1}$ to be sub-exponential in Assumption 4.1. This extension is necessary because many examples of generalized linear models (e.g., exponential regression and Poisson regression) satisfy the sub-exponential condition but not the sub-Gaussian condition. We also allow $C_{n}$ to possibly grow with $n$. Specifically, when $X_{i j}$ is uniformly bounded, $C_{n}$ is a positive constant. When $X_{i j}$ is sub-Gaussian, then $\max _{1 \leq i \leq n, 1 \leq j \leq d}\left|X_{i j}\right|=O_{p}\left(\{\log (n d)\}^{1 / 2}\right)$. Assumption 4.2 requires a similar sparsity condition, and allows $C_{n}$ to increase with $n$. Part (1) and (4) of Assumption 4.3 are identical to Assumption 3.6. Part (2) is a mild condition, stating that the regression effect in the outcome model is bounded. The third order differentiability of $b(u)$ holds for most generalized linear models. Part (3) is a technical condition. To analyze the estimator $\widehat{\beta}$ in Step 2 , we need to control $w_{1}(u, v)$ and $w_{1}^{\prime}(u, v)$ in a small neighborhood of the true values. This condition holds for two important examples $w_{1}(u, v)=\pi(v)$ and $w_{1}(u, v)=b^{\prime \prime}(u)$. The former corresponds to example (a) in Section 2.2, and the latter represents the generalization of example (b) to the generalized linear models.

To study the performance of our estimator under misspecified models, as done in Sections 3.3 and 3.4, we define the least false parameters as,

$$
\begin{aligned}
& \beta^{o}=\operatorname{argmax} \mathbb{E}\left[\int_{0}^{\beta^{\top} X_{i}}\left\{\frac{T_{i}}{\pi(u)}-1\right\} w_{1}\left(X_{i}^{\top} \alpha^{*}, u\right) d u\right], \\
& \alpha^{o}=\operatorname{argmin} \mathbb{E}\left\{\frac{T_{i} w_{2}\left(\beta^{* \top} X_{i}\right)}{a(\phi)}\left\{Y_{i} \alpha^{\top} X_{i}-b\left(\alpha^{\top} X_{i}\right)\right\}\right\} .
\end{aligned}
$$


The following main theorem in this section establishes the asymptotic normality of $\widehat{\mu}_{1}$ when the outcome variable follows a generalized linear model. When analyzing the theoretical properties under model misspecification, we replace $\alpha^{*}$ and $\beta^{*}$ in all assumptions with $\alpha^{o}$ and $\beta^{o}$.

Theorem 4.4 (Asymptotic Properties under the Generalized Linear Models). Suppose that Assumptions 3.1, 3.2, 3.5, 4.1, 4.2, and 4.3 hold, and the tuning parameters satisfy $\lambda_{0} \asymp \lambda \asymp \lambda^{\prime} \asymp$ $\{\log (d \vee n) / n\}^{1 / 2}$.

(1) Assume that both the propensity score model (2.2) and the outcome model (4.1) are correctly specified. Then the estimator $\widehat{\mu}_{1}$ with any weight functions $w_{1}(u, v)$ and $w_{2}(u)$ satisfies

$$
\widehat{\mu}_{1}-\mu_{1}^{*}=\frac{1}{n} \sum_{i=1}^{n}\left[\frac{T_{i}}{\pi_{i}^{*}}\left\{Y_{i}(1)-b^{\prime}\left(\alpha^{* \top} X_{i}\right)\right\}+b^{\prime}\left(\alpha^{* \top} X_{i}\right)-\mu_{1}^{*}\right]+o_{p}\left(n^{-1 / 2}\right),
$$

and $\widehat{\mu}_{1}$ achieves the same semiparametric efficiency bound.

(2) Assume that the outcome model (4.1) is correctly specified, but the propensity score model (2.2) is misspecified. If we choose $w_{1}(u, v)=b^{\prime \prime}(u)$, then for any $w_{2}(u)$ we have

$$
\widehat{\mu}_{1}-\mu_{1}^{*}=\frac{1}{n} \sum_{i=1}^{n}\left[\frac{T_{i}}{\pi\left(X_{i}^{\top} \beta^{o}\right)}\left\{Y_{i}(1)-b^{\prime}\left(\alpha^{* \top} X_{i}\right)\right\}+b^{\prime}\left(\alpha^{* \top} X_{i}\right)-\mu_{1}^{*}\right]+o_{p}\left(n^{-1 / 2}\right) .
$$

(3) Suppose that the propensity score model (2.2) is correctly specified, but the outcome model (4.1) is misspecified. If we set $w_{2}(u)=\pi^{\prime}(u) / \pi^{2}(u)$, then for any $w_{1}(u, v)$ we have

$$
\widehat{\mu}_{1}-\mu_{1}^{*}=\frac{1}{n} \sum_{i=1}^{n}\left[\frac{T_{i}}{\pi_{i}^{*}}\left\{Y_{i}(1)-b^{\prime}\left(\alpha^{o \top} X_{i}\right)\right\}+b^{\prime}\left(\alpha^{o \top} X_{i}\right)-\mu_{1}^{*}\right]+o_{p}\left(n^{-1 / 2}\right) .
$$

Part (1) of Theorem 4.4 is the extension of Theorem 3.7 to the generalized linear models. Under the correct model specification, the asymptotic normality of $\widehat{\mu}_{1}$ holds for any weight functions $w_{1}(u, v)$ and $w_{2}(u)$ that satisfy Assumption 4.3. This result agrees with the theory of AIPW estimators in Belloni et al. (2013a) and Farrell (2015). Unlike the existing work, parts (2) and (3) provide novel results on the asymptotic normality of $\widehat{\mu}_{1}$ when either the propensity score model or the outcome model is misspecified. Similar to Propositions 3.12 and 3.13, these results hold only if particular forms of $w_{1}(u, v)$ and $w_{2}(u)$ are chosen to remove the bias from model misspecification. In particular, we use the weight $w_{1}(u, v)=b^{\prime \prime}(u)$ in part (2), which requires the knowledge of $\alpha^{*}$ in the outcome model. This explains why Step 1 is needed. Since part (2) holds for any weight function $w_{2}(u)$, the estimator $\widehat{\mu}_{1}$ remains asymptotically normal even if we skip Step 3 and replace $\widetilde{\alpha}$ in Step 4 with $\widehat{\alpha}$ in Step 1. Similarly, part (3) holds for any weight function $w_{1}(u, v)$. Thus, if we set $w_{2}(u)=\pi^{\prime}(u) / \pi^{2}(u)$ and $w_{1}(u, v)=\pi(v)$, we may skip Step 1 of our algorithm and the same result in part (3) still applies.

Similar to Remark 3.14, the proposed estimator $\widehat{\mu}_{1}$ when $w_{1}(u, v)=b^{\prime \prime}(u)$ and $w_{2}(u)=$ $\pi^{\prime}(u) / \pi^{2}(u)$ is asymptotically normal provided that either the propensity score model or the out- 
come model is correctly specified. This estimator does not require to know which of the two models is correct, and therefore is recommended for practical use.

\section{Simulation Studies}

In this section, we conduct simulation studies to evaluate the finite sample performance of the proposed HD-CBPS methodology. We consider the following data generating processes. First, we generate the $d$ dimensional covariate $X_{i} \sim N(0, \Sigma)$ where $\Sigma_{j k}=\rho^{|j-k|}$ with $\rho=1 / 2$. We generate the binary treatment $T_{i}$ using the logistic regression model of the form, $\pi\left(X_{i}\right)=1-$ $1 /\left\{1+\exp \left(-X_{i 1}+X_{i 2} / 2-X_{i 3} / 4-X_{i 4} / 10-X_{i 5} / 10+X_{i 6} / 10\right)\right\}$. For the potential outcomes, we consider both linear and logistic regression models as specified later. The observed outcome is $Y_{i}=Y_{i}(1) T_{i}+Y_{i}(0)\left(1-T_{i}\right)$.

The simulation is repeated 200 times under each setting. Throughout the simulation studies, whenever possible, we compare our method (HD-CBPS) to the approximate residual balancing (RB) method (Athey et al., 2016), the regularized AIPW (AIPW) method (Farrell, 2015; Belloni et al., 2013a) and the double selection (Belloni et al., 2014). For the sake of comparison, we use the Lasso penalty in both HD-CBPS and AIPW methods, and all tuning parameters are determined by the 5 fold cross-validation. The weight functions in our method are chosen according to Remark 3.14. For the RB method, we use the default values of the tuning parameters in the $\mathrm{R}$ package balanceHD. The double selection method is implemented using the $\mathrm{R}$ package hdm.

We first consider the setting, in which the potential outcomes are generated from the linear regression models:

$$
\begin{aligned}
& Y_{i}(1)=2+0 \cdot 137\left(X_{i 5}+X_{i 6}+X_{i 7}+X_{i 8}\right)+\epsilon_{1 i}, \\
& Y_{i}(0)=1+0 \cdot 291\left(X_{i 5}+X_{i 6}+X_{i 7}+X_{i 8}+X_{i 9}+X_{i 10}\right)+\epsilon_{0 i},
\end{aligned}
$$

where $\epsilon_{1 i}$ and $\epsilon_{0 i}$ are independent standard normal random variables. Under this setting, we consider the following four scenarios. In the first scenario, we assume that the propensity score and outcome models are correctly specified. In the second scenario, the outcome models are correctly specified but the propensity score model is misspecified. We use the transformed variables, $X_{m i s}=$ $\left\{\exp \left(X_{1} / 2\right), X_{2} /\left\{1+\exp \left(X_{1}\right)\right\}+10,\left(X_{1} X_{3} / 25+0 \cdot 6\right)^{3},\left(X_{2}+X_{4}+20\right)^{2}, X_{6}, \exp \left(X_{6}+X_{7}\right), X_{9}^{2}, X_{7}^{3}-\right.$ $\left.20, X_{9}, \cdots X_{d}\right\}$ to generate the treatment but the original variables $X$ to generate the outcome variables. In the third scenario, the propensity score model is correctly specified but the outcome models are misspecified. We use the same transformed variables $X_{m i s}$ to generate the outcomes but the original variables $X$ to generate the treatment. Finally, we consider a scenario, in which both the outcome and propensity score models are misspecified using the transformed covariates. This model misspecification follows the work of Kang and Schafer (2007) who evaluated the empirical performance of the AIPW estimator in low-dimensional settings.

Table 1 shows the bias, standard error, standardized root mean squared error (RMSE) $\left\{\mathbb{E}(\widehat{\mu}-\mu)^{2}\right\}^{1 / 2} / \mu$, coverage probability of $95 \%$ confidence intervals, and their length for the estimation of the average treatment effect under the four scenarios. We focus on the comparison in the high-dimensional setting with $d=1000,2000$ and sample size $n=500,1000$. Some additional simulation studies for 
smaller $d$ is deferred to the supplementary materials. Table 1 shows that the proposed method tends to have smaller RMSE in most scenarios. More importantly, as seen in scenarios (2) and (3), the fact that the HD-CBPS has an accurate coverage probability under model misspecification provides empirical support for the robustness property established in Propositions 3.12 and 3.13. In contrast, the AIPW estimator has a significant bias under scenarios (2) and (3). As a result, its coverage probability is below 0.95 in most cases. The other two methods, i.e., RB and double selection, perform reasonably well under model misspecification. But their confidence intervals tend to be wider than the proposed method.

We also consider the simulation with logistic outcome models. When the outcome variable is binary, RB is not directly applicable. Thus, we only compare our method with the regularized AIPW and the double selection method. The simulation results illustrate the same conclusion. Due to the space constraint, we defer the details to the supplementary materials. The supplementary materials also contain more extensive numerical results including the simulations under different data generating processes, non-sparse models, comparisons with many other estimators (e.g., normalized Horvitz-Thompson estimator, calibrated likelihood (Tan, 2010), targeted maximum likelihood estimator (Van der Laan and Rose, 2011), IPW, and standard CBPS estimators) under a moderate dimension, and sensitivity analysis with respect to the choice of tuning parameters.

In summary, the proposed HD-CBPS estimator tends to have a smaller mean squared error, is more robust to model misspecification, and exhibits accurate coverage probability in finite samples. Our results are consistent with the empirical findings of Imai and Ratkovic (2014) and Fan et al. (2016) that covariate balancing tends to outperform the AIPW estimator in low-dimensional settings. Our simulation studies imply that the same conclusion appears to hold in high-dimensional settings.

\section{Empirical Illustration}

For empirical illustration, we consider a dataset obtained from the first two waves of Jennings' and Niemi's Political Socialization Panel Study, which is originally analyzed by Kam and Palmer (2008). One purpose of this study is to understand the effect of higher education on political participation. The dataset consists of 1,051 randomly selected high school seniors in the class of 1965. The information about each sample is collected via in-person interviews in the first wave of the study, which we treat as pre-treatment covariates. The second wave of the study conducted in 1973 collects the outcome variable, political participation, as well as the dichotomous treatment variable, college attendance.

For the purpose of comparison, we follow the original study and use 81 pre-treatment covariates, which include gender, race, club participation, and academic performance. Since many of the covariates are categorical variables with more than two levels, we create an indicator variable that represents each level. Therefore, a total of 204 pre-treatment variables are used in the propensity score and outcome models. The outcome variable represents an index of adult political participation, which is equal to the sum of eight acts including the turnout in the 1972 presidential election, attending campaign rallies, making a donation to a campaign, and displaying a campaign button 
and bumper sticker. Since this variable takes an integer value ranging from zero to eight, we use the binomial logistic regression for the outcome model. The propensity score model is assumed to be the logistic regression. We then estimate both the ATE and ATT of college attendace on political participation (the number of treated observations is 675).

We apply five methods to analyze this dataset, the proposed HD-CBPS methodology, the regularized AIPW method (Farrell, 2015), the original CBPS method (Imai and Ratkovic, 2014), the AIPW method without regularization (AIPW-NR) (Robins et al., 1994) and the IPW estimator with the regularized logistic regression (IPW). The estimation procedures for the first two methods are identical to those described in the simulation studies. For the original CBPS methodology, it is designed for the linear outcome model, which does not provide an ideal balance of pre-treatment variables. In addition, we use the bootstrap method to approximate the standard error of the estimator based on the CBPS and IPW methods.

The results are shown in Table 2. The HD-CBPS, AIPW, IPW and CBPS methods imply that the overall ATE of college education on political participation is positive and statistically significant while AIPW-NR yields a smaller estimate with a larger standard error. The ATE estimates and their associated standard errors based on the regularized methods (i.e., HD-CBPS, IPW and AIPW) are quite similar to each other. These ATE estimates are, however, smaller than that of CBPS and greater than that of AIPW-NR. More importantly, for both the ATE and the ATT, the regularized estimate HD-CBPS has much smaller standard errors than CBPS and AIPW-NR. There are at least two reasons for this difference in standard errors. First, as shown in Section 4, the HD-CBPS methodology uses a different covariate balancing estimating equation than CBPS when the outcome model is nonlinear, achieving the semiparametric efficiency bound. Second, the original CBPS methodology tends to be unstable when balancing a large number of covariates (204 in this case). Thus, the proposed HD-CBPS method improves the existing covariate balancing methods when the outcome model belongs to the class of generalized linear models and the number of covariates is large.

We also apply the HD-CBPS, AIPW, IPW and AIPW-NR methods to the subsample of whites separately. Among a total of 1,051 respondents, there are 966 white respondents. In this case, the CBPS method does not converge, and therefore the estimate is unavailable. The results appear at the last row of Table 2. Again, the estimates of the two regularized methods are similar so are the standard errors. However, the AIPW-NR methodology shows very large variance, mainly because the maximum likelihood estimate of propensity score tends to be very unstable when the number of covariates is large.

\section{Discussion}

There are several future directions that are worthy of further investigation. First, it is important to extend these high-dimensional causal inference methods to non-binary treatment regimes, including continuous treatment and dynamic treatment regimes. Second, we plan to further study the effect of tuning parameters on statistical inference. In numerical experiments, the tuning parameters are chosen by the cross-validation, which leads to reasonable finite sample results. Based on the 
sensitivity analysis, the results appear to be stable with respect to a small perturbation of tuning parameters. One interesting problem is to formally justify the validity of the inference based on the cross-validated estimators. The current research on the cross-validated Lasso estimator only guarantees a slow rate of convergence when the error is sub-Gaussian (e.g., Chetverikov et al. (2016) and the references therein), which is not sufficient to control the remainder terms in the proof of Theorem 3.7. Further theoretical development is needed to address this important problem.

\section{Acknowledgement}

We thank Jamie Robins for the insightful discussion.

\section{Supplementary material}

Supplementary material includes proofs of the theoretical results, additional simulation results and further technical details.

\section{References}

Athey, S., Imbens, G. W. and Wager, S. (2016). Efficient inference of average treatment effects in high dimensions via approximate residual balancing. arXiv preprint arXiv:1604.07125 .

BAng, H. and Robins, J. M. (2005). Doubly robust estimation in missing data and causal inference models. Biometrics 61 962-973.

Belloni, A., Chernozhukov, V., Fernández-Val, I. and Hansen, C. (2013a). Program evaluation with high-dimensional data. arXiv preprint arXiv:1311.2645 .

Belloni, A., Chernozhukov, V. and Hansen, C. (2014). Inference on treatment effects after selection among high-dimensional controls. The Review of Economic Studies 81 608-650.

Belloni, A., Chernozhukov, V. and Wei, Y. (2016). Post-selection inference for generalized linear models with many controls. Journal of Business 83 Economic Statistics 16 606-619.

Belloni, A., Chernozhukov, V. et Al. (2013b). Least squares after model selection in highdimensional sparse models. Bernoulli 19 521-547.

Bickel, P. J., Ritov, Y. and Tsybakov, A. B. (2009). Simultaneous analysis of lasso and dantzig selector. The Annals of Statistics 37 1705-1732.

Bühlmann, P. and van de Geer, S. (2015). High-dimensional inference in misspecified linear models. Electronic Journal of Statistics 9 1449-1473.

Busso, M., DiNardo, J. and McCrary, J. (2014). New evidence on the finite sample properties of propensity score reweighting and matching estimators. Review of Economics and Statistics 96 885-897. 
CAI, T. T. and Guo, Z. (2015). Confidence intervals for high-dimensional linear regression: Minimax rates and adaptivity. arXiv preprint arXiv:1506.05539 .

Cao, W., Tsiatis, A. A. and Davidian, M. (2009). Improving efficiency and robustness of the doubly robust estimator for a population mean with incomplete data. Biometrika asp033.

Chan, K. C. G., Yam, S. C. P. and Zhang, Z. (2016). Globally efficient nonparametric inference of average treatment effects by empirical balancing calibration weighting. Journal of the Royal Statistical Society, Series B, Methodological 78 673-700.

Chernozhukov, V., Chetverikov, D., Demirer, M., Duflo, E., Hansen, C. and Newey, W. (2016). Double machine learning for treatment and causal parameters. arXiv preprint arXiv:1608.00060, 2016 .

Chetverikov, D., Liao, Z. and Chernozhukov, V. (2016). On cross-validated lasso. arXiv preprint arXiv:1605.02214.

Dukes, O., Avagyan, V. and Vansteelandt, S. (2018). High-dimensional doubly robust tests for regression parameters. arXiv preprint arXiv:1805.06714.

Fan, J., Imai, K., LiU, H., Ning, Y. and YANG, X. (2016). Improving covariate balancing propensity score: A doubly robust and efficient approach. Technical Report .

FAN, J. and Li, R. (2001). Variable selection via nonconcave penalized likelihood and its oracle properties. Journal of the American Statistical Association 96 1348-1360.

FARRELL, M. H. (2015). Robust inference on average treatment effects with possibly more covariates than observations. Journal of Econometrics 189 1-23.

Fong, C., Ratkovic, M. and Imai, K. (2018). CBPS: R package for covariate balancing propensity score. available at the Comprehensive R Archive Network (CRAN). https: //CRAN.R-project. org/package=CBPS.

Graham, B. S., Pinto, C. and Egel, D. (2012). Inverse probability tilting for moment condition models with missing data. Review of Economic Studies 79 1053-1079.

HAHN, J. (1998). On the role of the propensity score in efficient semiparametric estimation of average treatment effects. Econometrica 315-331.

Hahn, J. (2004). Functional restriction and efficiency in causal inference. Review of Economics and Statistics 86 73-76.

Hainmueller, J. (2012). Entropy balancing for causal effects: Multivariate reweighting method to produce balanced samples in observational studies. Political Analysis 20 25-46.

HÁJEK, J. (1971). Foudations of Statistical Inference (eds. V. Godambe and D. Sprott), chap. Comment on "An essay on the logical foundations of survey sampling, part one" by D. Basu. Holt, Rinehart, and Winston, Toronto, 236. 
Horvitz, D. and Thompson, D. (1952). A generalization of sampling without replacement from a finite universe. Journal of the American Statistical Association 47 663-685.

Imai, K. and Ratkovic, M. (2014). Covariate balancing propensity score. J. R. Stat. Soc. Ser. B. Stat. Methodol. 76 243-263.

ImAI, K. and van Dyk, D. A. (2004). Causal inference with general treatment regimes: Generalizing the propensity score. Journal of the American Statistical Association 99 854-866.

Imbens, G. W. (2000). The role of the propensity score in estimating dose-response functions. Biometrika 87 706-710.

Imbens, G. W., Newey, W. K. and Ridder, G. (2005). Mean-square-error calculations for average treatment effects .

Javanmard, A. and Montanari, A. (2013). Confidence intervals and hypothesis testing for high-dimensional regression. arXiv preprint arXiv:1306.3171 .

Kam, C. D. and Palmer, C. L. (2008). Reconsidering the effects of education on political participation. The Journal of Politics $\mathbf{7 0} 612-631$.

Kang, J. D. and Schafer, J. L. (2007). Demystifying double robustness: A comparison of alternative strategies for estimating a population mean from incomplete data. Statistical science $523-539$.

Lunceford, J. K. and Davidian, M. (2004). Stratification and weighting via the propensity score in estimation of causal treatment effects: a comparative study. Statistics in Medicine $\mathbf{2 3}$ $2937-2960$.

Ning, Y. and LiU, H. (2017). A general theory of hypothesis tests and confidence regions for sparse high dimensional models. The Annals of Statistics 45 158-195.

Robins, J., Sued, M., Lei-Gomez, Q. and Rotnitzky, A. (2007). Comment: Performance of double-robust estimators when" inverse probability" weights are highly variable. Statistical Science 22 544-559.

Robins, J. M., Rotnitzky, A. and Zhao, L. P. (1994). Estimation of regression coefficients when some regressors are not always observed. Journal of the American Statistical Association $89846-866$.

Rosenbaum, P. R. and Rubin, D. B. (1983). The central role of the propensity score in observational studies for causal effects. Biometrika 70 41-55.

Rubin, D. B. (1990). Comments on "On the application of probability theory to agricultural experiments. Essay on principles. Section 9" by J. Splawa-Neyman translated from the Polish and edited by D. M. Dabrowska and T. P. Speed. Statistical Science 5 472-480. 
Rubin, D. B. (2006). Matched Sampling for Causal Effects. Cambridge University Press, Cambridge.

Rubin, D. B. (2008). For objective causal inference, design trumps analysis. Annals of Applied Statistics 2 808-840.

Schneeweiss, S., Rassen, J. A., Glynn, R. J., Avorn, J., Mogun, H. and Brookhart, M. A. (2009). High-dimensional propensity score adjustment in studies of treatment effects using health care claims data. Epidemiology 20 512-522.

TAN, Z. (2010). Bounded, efficient and doubly robust estimation with inverse weighting. Biometrika $97661-682$.

TAN, Z. (2017). Regularized calibrated estimation of propensity scores with model misspecification and high-dimensional data. arXiv preprint arXiv:1710.08074 .

TAN, Z. (2018). Model-assisted inference for treatment effects using regularized calibrated estimation with high-dimensional data. arXiv preprint arXiv:1801.09817 .

Tibshirani, R. (1996). Regression shrinkage and selection via the lasso. Journal of the Royal Statistical Society. Series B (Methodological) 267-288.

van de Geer, S., Bühlmann, P., Ritov, Y. and Dezeure, R. (2014). On asymptotically optimal confidence regions and tests for high-dimensional models. The Annals of Statistics 11661202.

VAn Der LaAn, M. J. and Rose, S. (2011). Targeted learning: causal inference for observational and experimental data. Springer Science \& Business Media.

Vermeulen, K. and Vansteelandt, S. (2015). Bias-reduced doubly robust estimation. Journal of the American Statistical Association 110 1024-1036.

Wedderburn, R. W. (1974). Quasi-likelihood functions, generalized linear models, and the gauss-newton method. Biometrika 61 439-447.

Zhang, C.-H. and Zhang, S. S. (2014). Confidence intervals for low dimensional parameters in high dimensional linear models. Journal of the Royal Statistical Society: Series B (Statistical Methodology) $\mathbf{7 6} 217-242$.

ZhaO, P. and Yu, B. (2006). On model selection consistency of lasso. Journal of Machine learning research 7 2541-2563.

Zнао, Q. (2016). Covariate balancing propensity score by tailored loss functions. arXiv preprint arXiv:1601.05890 .

Zubizarreta, J. R. (2015). Stable weights that balance covariates for estimation with incomplete outcome data. Journal of the American Statistical Association 110 910-922. 
Table 1: Bias, standard error (Std Err), standardized root-mean-squared error (RMSE), coverage probability of $95 \%$ confidence intervals (Coverage), and length of $95 \%$ confidence intervals (CI length) for the estimation of the ATE. Four methods - high-dimensional CBPS, approximate residual balancing, regularized augmented inverse probability weighting, and double selection - are compared.

\begin{tabular}{|c|c|c|c|c|c|c|c|c|}
\hline \multirow[t]{2}{*}{$n=500$} & \multicolumn{4}{|c|}{$d=1000$} & \multicolumn{4}{|c|}{$d=2000$} \\
\hline & HD-CBPS & $\mathrm{RB}$ & AIPW & D-SELECT & HD-CBPS & $\mathrm{RB}$ & AIPW & D-SELECT \\
\hline \multicolumn{9}{|c|}{ (1) Both models are correct } \\
\hline Bias & -0.0026 & $-0 \cdot 0017$ & $-0 \cdot 0498$ & $-0 \cdot 0910$ & -0.0595 & $-0 \cdot 0580$ & $-0 \cdot 1200$ & $-0 \cdot 0397$ \\
\hline Std Err & $0 \cdot 0936$ & $0 \cdot 1074$ & $0 \cdot 0926$ & $0 \cdot 0979$ & $0 \cdot 1061$ & $0 \cdot 1155$ & $0 \cdot 1011$ & $0 \cdot 1279$ \\
\hline RMSE & $0 \cdot 0936$ & $0 \cdot 1074$ & $0 \cdot 1052$ & $0 \cdot 1337$ & $0 \cdot 1216$ & $0 \cdot 1292$ & $0 \cdot 1569$ & $0 \cdot 1334$ \\
\hline Coverage & $0 \cdot 965$ & $0 \cdot 930$ & $0 \cdot 915$ & $0 \cdot 890$ & $0 \cdot 910$ & $0 \cdot 910$ & $0 \cdot 855$ & 0.945 \\
\hline CI length & $0 \cdot 3867$ & $0 \cdot 4231$ & $0 \cdot 3775$ & $0 \cdot 4294$ & $0 \cdot 3862$ & $0 \cdot 4359$ & $0 \cdot 3731$ & $0 \cdot 5034$ \\
\hline \multicolumn{9}{|c|}{ (2) Propensity score model is misspecified } \\
\hline Bias & $-0 \cdot 0120$ & $-0 \cdot 0303$ & $-0 \cdot 1078$ & $-0 \cdot 0782$ & $-0 \cdot 0446$ & $-0 \cdot 0685$ & $-0 \cdot 1234$ & $-0 \cdot 0357$ \\
\hline Std Err & $0 \cdot 0984$ & $0 \cdot 1153$ & $0 \cdot 0963$ & $0 \cdot 1034$ & $0 \cdot 0924$ & $0 \cdot 1041$ & $0 \cdot 0921$ & $0 \cdot 1214$ \\
\hline RMSE & $0 \cdot 0991$ & $0 \cdot 1193$ & $0 \cdot 1446$ & $0 \cdot 1296$ & $0 \cdot 1025$ & $0 \cdot 1246$ & $0 \cdot 1540$ & $0 \cdot 1265$ \\
\hline Coverage & 0.965 & $0 \cdot 945$ & $0 \cdot 815$ & $0 \cdot 905$ & $0 \cdot 930$ & $0 \cdot 910$ & $0 \cdot 740$ & $0 \cdot 940$ \\
\hline CI length & $0 \cdot 3864$ & $0 \cdot 4431$ & $0 \cdot 3732$ & $0 \cdot 4227$ & $0 \cdot 3839$ & $0 \cdot 4382$ & $0 \cdot 3702$ & $0 \cdot 5023$ \\
\hline \multicolumn{9}{|c|}{ (3) Outcome model is misspecified } \\
\hline Bias & $-0 \cdot 0034$ & $-0 \cdot 0321$ & $-0 \cdot 0562$ & $-0 \cdot 0991$ & $-0 \cdot 0317$ & -0.0572 & $-0 \cdot 1215$ & $-0 \cdot 0443$ \\
\hline Std Err & $0 \cdot 0917$ & $0 \cdot 0982$ & $0 \cdot 0914$ & $0 \cdot 1023$ & $0 \cdot 0944$ & $0 \cdot 0992$ & $0 \cdot 0921$ & $0 \cdot 1026$ \\
\hline RMSE & $0 \cdot 0917$ & $0 \cdot 1033$ & $0 \cdot 1072$ & $0 \cdot 1424$ & $0 \cdot 0995$ & $0 \cdot 1145$ & $0 \cdot 1525$ & $0 \cdot 1118$ \\
\hline Coverage & $0 \cdot 960$ & $0 \cdot 960$ & $0 \cdot 905$ & $0 \cdot 845$ & $0 \cdot 950$ & 0.955 & $0 \cdot 770$ & 0.945 \\
\hline CI length & $0 \cdot 3874$ & $0 \cdot 4292$ & $0 \cdot 3815$ & $0 \cdot 4327$ & $0 \cdot 3890$ & $0 \cdot 4403$ & $0 \cdot 3728$ & $0 \cdot 4261$ \\
\hline \multicolumn{9}{|c|}{ (4) Both models are misspecified } \\
\hline Bias & $-0 \cdot 0547$ & $-0 \cdot 1201$ & $-0 \cdot 1873$ & $-0 \cdot 1005$ & $-0 \cdot 0243$ & $-0 \cdot 0599$ & $-0 \cdot 1393$ & $-0 \cdot 0518$ \\
\hline Std Err & $0 \cdot 1106$ & $0 \cdot 1038$ & $0 \cdot 0903$ & $0 \cdot 0950$ & $0 \cdot 0969$ & $0 \cdot 1060$ & $0 \cdot 0921$ & $0 \cdot 0965$ \\
\hline RMSE & $0 \cdot 1234$ & $0 \cdot 1588$ & $0 \cdot 2079$ & $0 \cdot 1383$ & $0 \cdot 0999$ & $0 \cdot 1218$ & $0 \cdot 1670$ & $0 \cdot 1095$ \\
\hline Coverage & $0 \cdot 890$ & $0 \cdot 815$ & $0 \cdot 775$ & $0 \cdot 875$ & $0 \cdot 940$ & $0 \cdot 940$ & $0 \cdot 720$ & 0.950 \\
\hline CI length & $0 \cdot 3994$ & $0 \cdot 4586$ & $0 \cdot 3790$ & $0 \cdot 4333$ & $0 \cdot 3948$ & $0 \cdot 4545$ & $0 \cdot 3781$ & $0 \cdot 4334$ \\
\hline \multirow[t]{2}{*}{$n=1000$} & \multicolumn{4}{|c|}{$d=1000$} & \multicolumn{4}{|c|}{$d=2000$} \\
\hline & HD-CBPS & $\mathrm{RB}$ & AIPW & D-SELECT & HD-CBPS & $\mathrm{RB}$ & AIPW & D-SELECT \\
\hline \multicolumn{9}{|c|}{ (1) Both models are correct } \\
\hline Bias & $-0 \cdot 0233$ & $-0 \cdot 0234$ & $-0 \cdot 0814$ & $-0 \cdot 0476$ & $0 \cdot 0199$ & $0 \cdot 0186$ & $-0 \cdot 0056$ & $0 \cdot 0249$ \\
\hline Std Err & $0 \cdot 0669$ & $0 \cdot 0777$ & $0 \cdot 0647$ & $0 \cdot 0690$ & $0 \cdot 0659$ & $0 \cdot 07476$ & $0 \cdot 0654$ & $0 \cdot 0757$ \\
\hline RMSE & $0 \cdot 0695$ & $0 \cdot 0729$ & $0 \cdot 0678$ & $0 \cdot 0839$ & $0 \cdot 0689$ & $0 \cdot 0769$ & $0 \cdot 0657$ & $0 \cdot 0797$ \\
\hline Coverage & $0 \cdot 955$ & $0 \cdot 940$ & $0 \cdot 905$ & $0 \cdot 920$ & $0 \cdot 940$ & $0 \cdot 935$ & $0 \cdot 950$ & 0.955 \\
\hline CI length & $0 \cdot 2828$ & $0 \cdot 3010$ & $0 \cdot 2700$ & $0 \cdot 2978$ & $0 \cdot 2746$ & $0 \cdot 2979$ & $0 \cdot 2697$ & $0 \cdot 3187$ \\
\hline \multicolumn{9}{|c|}{ (2) Propensity score model is misspecified } \\
\hline Bias & $-0 \cdot 0297$ & $-0 \cdot 0455$ & $-0 \cdot 0931$ & $-0 \cdot 0362$ & $0 \cdot 0164$ & $0 \cdot 0135$ & $-0 \cdot 0137$ & $0 \cdot 0116$ \\
\hline Std Err & $0 \cdot 0607$ & $0 \cdot 0694$ & $0 \cdot 0605$ & $0 \cdot 0665$ & $0 \cdot 0662$ & $0 \cdot 0758$ & $0 \cdot 0659$ & $0 \cdot 0846$ \\
\hline RMSE & $0 \cdot 0671$ & $0 \cdot 0842$ & $0 \cdot 1105$ & $0 \cdot 0757$ & $0 \cdot 0682$ & $0 \cdot 0770$ & $0 \cdot 0673$ & $0 \cdot 0854$ \\
\hline Coverage & $0 \cdot 970$ & $0 \cdot 930$ & $0 \cdot 855$ & 0.930 & $0 \cdot 940$ & 0.955 & 0.955 & 0.935 \\
\hline CI length & $0 \cdot 2801$ & $0 \cdot 3040$ & $0 \cdot 2694$ & $0 \cdot 2960$ & $0 \cdot 2746$ & $0 \cdot 2987$ & $0 \cdot 2697$ & $0 \cdot 3381$ \\
\hline \multicolumn{9}{|c|}{ (3) Outcome model is misspecified } \\
\hline Bias & $-0 \cdot 0222$ & $-0 \cdot 0229$ & $-0 \cdot 0821$ & $-0 \cdot 0436$ & $-0 \cdot 0062$ & $-0 \cdot 0026$ & $-0 \cdot 0517$ & 0.0262 \\
\hline Std Err & $0 \cdot 0670$ & $0 \cdot 0699$ & $0 \cdot 0653$ & $0 \cdot 0671$ & $0 \cdot 0653$ & $0 \cdot 0709$ & $0 \cdot 0630$ & $0 \cdot 0669$ \\
\hline RMSE & $0 \cdot 0706$ & 0.0735 & $0 \cdot 1049$ & $0 \cdot 0800$ & $0 \cdot 0656$ & $0 \cdot 0709$ & $0 \cdot 0815$ & $0 \cdot 0718$ \\
\hline Coverage & $0 \cdot 960$ & $0 \cdot 960$ & $0 \cdot 890$ & 0.955 & 0.975 & $0 \cdot 970$ & 0.930 & 0.965 \\
\hline CI length & $0 \cdot 2842$ & $0 \cdot 3058$ & $0 \cdot 2709$ & $0 \cdot 3002$ & $0 \cdot 2848$ & $0 \cdot 3139$ & $0 \cdot 2726$ & $0 \cdot 2920$ \\
\hline \multicolumn{9}{|c|}{ (4) Both models are misspecified } \\
\hline Bias & $-0 \cdot 0157$ & $-0 \cdot 0072$ & $-0 \cdot 0504$ & $-0 \cdot 0366$ & $0 \cdot 0150$ & $0 \cdot 0009$ & $-0 \cdot 0635$ & $0 \cdot 0076$ \\
\hline Std Err & $0 \cdot 0701$ & $0 \cdot 0822$ & $0 \cdot 0721$ & $0 \cdot 0687$ & $0 \cdot 0613$ & $0 \cdot 0765$ & $0 \cdot 0598$ & $0 \cdot 0792$ \\
\hline RMSE & $0 \cdot 0718$ & $0 \cdot 0825$ & $0 \cdot 0880$ & $0 \cdot 0779$ & $0 \cdot 0631$ & $0 \cdot 0765$ & $0 \cdot 0872$ & $0 \cdot 0796$ \\
\hline Coverage & 0.945 & $0 \cdot 960$ & $0 \cdot 905$ & 0.925 & 0.990 & 0.960 & 0.905 & 0.950 \\
\hline CI length & $0 \cdot 2872$ & $0 \cdot 3117$ & $0 \cdot 2774$ & $0 \cdot 3046$ & $0 \cdot 2882$ & $0 \cdot 3281$ & $0 \cdot 2739$ & $0 \cdot 3426$ \\
\hline
\end{tabular}


Table 2: The estimated average effects of college attendance on political participation. The estimates based on the proposed HD-CBPS methodology are compared with those of the original CBPS estimator, the regularized augmented inverse probability weighted estimator (AIPW), the augmented inverse probability weighted estimator without regularization (AIPW-NR) and the inverse propensity score weighted estimator with the regularized logistic regression (IPW). Standard errors appear in parentheses.

\begin{tabular}{cccccc}
\hline & HD-CBPS & CBPS & AIPW & AIPW-NR & IPW \\
\hline \multirow{2}{*}{ Overall (ATE) } & $0 \cdot 8293$ & $1 \cdot 0163$ & $0 \cdot 8796$ & 0.4904 & 1.0666 \\
& $(0 \cdot 1247)$ & $(0 \cdot 2380)$ & $(0 \cdot 1043)$ & $(0 \cdot 6009)$ & $(0 \cdot 1588)$ \\
Overall (ATT) & $0 \cdot 8439$ & $1 \cdot 1232$ & & & \\
& $(0 \cdot 1420)$ & $(0 \cdot 3094)$ & & & \\
Whites (ATE) & $0 \cdot 8445$ & & 0.8977 & 0.1205 & 1.1371 \\
& $(0 \cdot 1279)$ & & $(0 \cdot 1089)$ & $(9 \cdot 4522)$ & $(0 \cdot 1548)$ \\
\hline
\end{tabular}

